\title{
Toward Energetically Consistent Ocean Models
}

\author{
CARSTEN EDEN AND LARS CZESCHEL \\ Institut für Meereskunde, Universität Hamburg, Hamburg, Germany \\ DIRK OLBERS \\ Alfred-Wegener-Institut für Polar und Meeresforschung, Bremerhaven, Germany
}

(Manuscript received 4 December 2013, in final form 25 August 2014)

\begin{abstract}
Possibilities to construct a realistic quasi-global ocean model in Boussinesq approximation with a closed energy cycle are explored in this study. In such a model, the energy related to the mean variables would interact with all parameterized forms of energy without any spurious energy sources or sinks. This means that the energy available for interior mixing in the ocean would be only controlled by external energy input from the atmosphere and the tidal system and by internal exchanges. In the current implementation of such a consistent model, however, numerical biases and sources due to the nonlinear equation of state violate energy conservation, resulting in an overall residual up to several percent. In three (approximately) consistent model versions with different scenarios of mesoscale eddy dissipation, the parameterized internal wave field provides between 2 and $3 \mathrm{TW}$ for interior mixing from the total external energy input of about $4 \mathrm{TW}$, such that a transfer between 0.3 and $0.4 \mathrm{TW}$ into mean potential energy contributes to drive the large-scale circulation in the model. In contrast, the wind work on the mean circulation contributes by about $1.8 \mathrm{TW}$ to the largescale circulation in all model versions. It is shown that the consistent model versions are more energetic than standard and inconsistent model versions and in better agreement with hydrographic observations.
\end{abstract}

\section{Introduction}

The dynamics of the ocean can be decomposed into three principal regimes: ${ }^{1}$ small-scale turbulence down to the smallest spatial and temporal scales, internal gravity waves over a wide range of spatial scales, and the geostrophically quasi-balanced motion ${ }^{2}$ at larger spatial and temporal scales (Fig. 1). Together with the external forcing, the interaction among the three dynamical regimes builds up the energy cycle of the ocean. The conversion of

\footnotetext{
${ }^{1}$ Since sound waves are believed to have no impact on the ocean circulation, it is safe to neglect them by applying the Boussinesq approximation.

${ }^{2}$ The near-surface "submesoscale" flow regime (e.g., Capet et al. 2008a)-although at larger Rossby number and with smaller equivalent Rossby radius than the flow in the stratified interior-is also balanced, and thus constitutes no new principal dynamical regime.
}

Corresponding author address: Carsten Eden, Institut für Meereskunde, Universität Hamburg, Bundesstr. 53, 20146 Hamburg, Germany.

E-mail: carsten.eden@zmaw.de. kinetic energy to internal energy (heat) by molecular friction takes place at the dissipative end of the smallscale turbulent kinetic energy (TKE) cascade, typically at the scale of centimeters in the interior of the ocean. The turbulent kinetic energy cascade toward this dissipative scale is fed by dissipative processes acting on the other dynamical regimes at larger scales. The nonlinear wave-wave interaction within the internal gravity wave field generates, for instance, an energy transfer to smaller scales or high vertical wavenumbers (e.g., Olbers 1974; Müller et al. 1986), and the resulting shear or convective instability, that is, the breaking of gravity waves, represents a key source of small-scale turbulence in the interior of the ocean (e.g., Munk 1981). Going to even larger scales, internal gravity waves can interact and can be generated by the geostrophically balanced meso- to large-scale circulation, for example, by flow over topography, ageostrophic instabilities, or by direct loss of geostrophic balance (Bell 1975; Ford et al. 2000; Molemaker et al. 2010).

On the other hand, kinetic energy dissipation at the smallest scale also involves enhanced molecular mixing of temperature and salinity, thus mixing of density (e.g., 


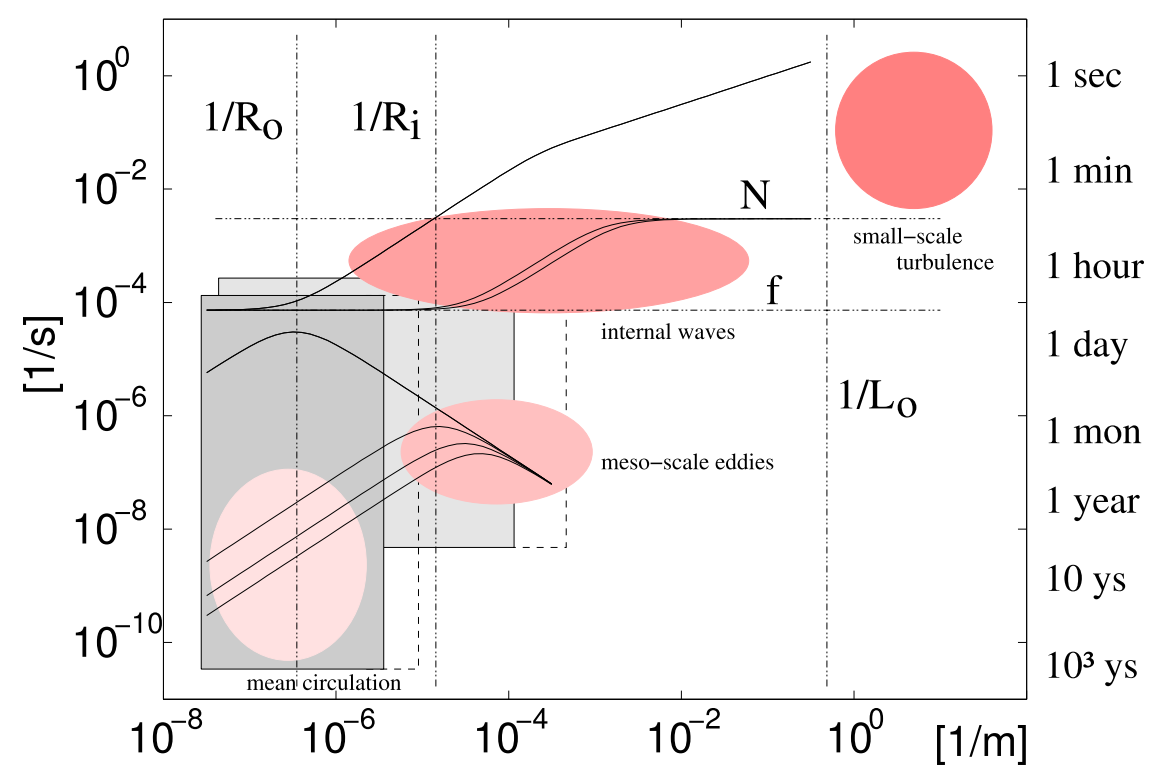

FIG. 1. Schematic of different dynamical regimes in the ocean as a function of wavenumber and frequency. Solid lines denote the dynamically most important linear wave solutions. Internal gravity waves for frequencies between the Coriolis frequency $f$ and the stability frequency $N$; planetary waves characterizing the balanced flow at much smaller frequencies and on spatial scale near or larger than the internal $R_{i}$ or barotropic $R_{o}$ Rossby radius. Also shown are surface gravity waves. Different solid lines denote different vertical modes or vertical wavenumbers. Small-scale turbulence is separated from the waves by the Ozmidov scale $L_{o}$. Gray boxes denote scales currently covered by non-eddy-resolving (dark) or eddy-permitting (light) ocean models. The expected gain in computer power in the next $10 \mathrm{yr}$ allows the extension of the ocean boxes by the dashed lines. Adopted from Olbers et al. (2012).

Osborn and Cox 1972). That means that a certain amount of the dissipated kinetic energy—given by the mixing efficiency-is spent for an exchange with the potential energy. This density mixing is known to be one of the drivers of the circulation in the ocean at the largest scale (Munk 1966), for instance, the meridional overturning circulation that is responsible for the advective ventilation of the abyssal ocean. Such interactions between the different dynamical regimes transfer large amounts of kinetic and potential energy and cascades the energy input by tidal and atmospheric forcing into the ocean to the dissipative scales. The internal wave field plays a central role in this cycle by linking different energy sources for mixing and dissipation, vertically and laterally over large distances. In ocean circulation models, most parts of the internal wave spectrum remain unresolved, in particular the part that is prone to shear instability and dissipation. The effect of unresolved dissipation of the internal gravity wave field is parameterized by the mixing of density with a prescribed diffusivity. Although this diffusivity is sometimes linked to resolved parameters such as the vertical stability or shear (e.g., Pacanowski and Philander 1981; Gargett 1984; Cummins et al. 1990), or to energy input into the internal wave field by tides (Jayne 2009; Olbers and Eden 2013), a consistent description of the energetics of the internal wave field including its interaction with all other dynamical regimes has not been considered so far in ocean modeling.

A similar argument holds for many parameterizations that are used today in ocean models; it is common for the dissipation of the (available) potential energy of the turbulent balanced flow in ocean models by an additional mesoscale eddy-driven advection velocity (Gent et al. 1995), the dissipation of resolved kinetic energy by harmonic or biharmonic lateral friction, and the dissipation of energy in bottom boundary layers. For all those processes, the kinetic and potential energy that is dissipated is simply lost instead of being transferred to the relevant connecting dynamical regime or to a different form of energy. On the other hand, at other places and for other parameterizations, this missing energy needs to be artificially created again. The most prominent example is the unaccounted supply of energy that is needed to mix the density in ocean models, but the same holds for almost any other parameterization and dynamical regime. In other words, current ocean models have no complete account on the energy cycle and are thus inconsistent in this way. It is the aim of the present 
study to resolve this inconsistency by connecting all the parameterizations in a state-of-the-art ocean model in an energetically consistent way.

In section 2, the configuration of the model is detailed, including the parameterizations that are used and how they are connected in a consistent way, that is, without any spurious energy sources or sinks. Section 3 shows some results of several experiments with the model, while section 4 discusses remaining imbalances in the model. Section 5 provides a summary.

\section{Model configuration}

In this section, we describe the parameterizations for the three principal dynamical regimes for our ocean model and how they can be connected to each other to obtain an energetically consistent ocean model. All parameterizations, which are detailed below in this section, are implemented in the Massachusetts Institute of Technology general circulation model (MITgcm) (Marshall et al.1997). The small-scale closure by Gaspar et al. (1990) was implemented by M. Losch (2011, personal communication); the closure for internal wave dissipation and the mesoscale eddy energy equation that adds to the already implemented parameterization by Gent and McWilliams (1990) in MITgcm was implemented by the authors. We use a realistic, non-eddy-resolving configuration $\left(1^{\circ} \times 1^{\circ}\right)$ with 110 vertical levels with thickness ranging from 10 to $83.3 \mathrm{~m}$ at the maximum depth of $5500 \mathrm{~m}$. The domain is quasi global, excludes the Arctic Ocean poleward of $80^{\circ} \mathrm{N}$, but includes the Southern Ocean equatorward of $80^{\circ} \mathrm{S}$. We use second-order advection schemes for tracer and momentum without implicit numerical mixing or dissipation. Dissipation of kinetic energy is only due to horizontal and vertical friction and we apply free-slip boundary conditions.

We use a monthly climatology of realistic forcing datasets for momentum and heat fluxes and a restoring boundary condition for surface salinity. The surface heat flux boundary condition follows Barnier et al. (1995) and is sometimes called a Haney-type surface boundary condition (Haney 1971). The forcing by Barnier et al. (1995) is based on the linearized bulk formulae and assumes an infinite heat reservoir of the atmosphere, but allows for an evolving ocean circulation. The restoring time scale for surface salinity is 90 days for the $10-\mathrm{m}$ thick surface grid box. There is no explicit sea ice model. In the case of surface temperatures below the freezing point, surface heat fluxes out of the ocean and salinity restoring (but not the momentum fluxes) are set to zero.

\section{a. Small-scale turbulence}

A variety of closures have been proposed to parameterize the effect of turbulence in the ocean on scales from a few centimeters to a couple of meters (e.g., Kantha and Clayson 2000). Although influenced by gravity and stratification, this kind of turbulence in the interior of the ocean is often called isotropic turbulence and in fact many predictions and results of the classical isotropic turbulence theories (e.g., Batchelor 1982) have been used and applied in practical closures. Many of those closures are based on equations of second- or higher-order quantities representative of the turbulent flow, with different attempts to close the hierarchy of the orders. An important second-order equation is the turbulent kinetic energy equation:

$$
\rho_{0} \frac{d \bar{E}_{\mathrm{tke}}}{d t}=-\partial_{z}(\text { fluxes })-\rho_{0} \overline{\mathbf{u}^{\prime} w^{\prime}} \partial_{z} \overline{\mathbf{u}}-g \overline{w^{\prime} \rho^{\prime}}-\nu \rho_{0} \overline{\left(\nabla \mathbf{u}^{\prime}\right)^{2}}
$$

where the second-order quantity $\bar{E}_{\mathrm{tke}}=\overline{\left(u^{\prime 2}+v^{\prime 2}+w^{\prime 2}\right)} / 2$ denotes the kinetic energy of the turbulent fluctuations of the flow (TKE), where $u^{\prime}, v^{\prime}, w^{\prime}$, and $\rho^{\prime}$ are deviations from the (statistical, time, or spatial) mean flow $\bar{u}, \bar{v}, \bar{w}$, and density (perturbation) $\bar{\rho}$ and where $\rho_{0}$ is the constant Boussinesq reference density. There are three source terms changing the TKE and a vertical divergence of fluxes containing a triple velocity correlation and pressurevelocity correlations, which couple both to higher-order quantities. A detailed derivation and discussion of Eq. (1) is provided by many textbooks, for example, Olbers et al. (2012); here, we only discuss the source terms in more detail, since we are concerned with the energy cycle, but note that the (parameterizations of the) flux terms also play an important role for a specific closure.

Downgradient (or negative) vertical eddy density fluxes $\overline{w^{\prime} \rho^{\prime}}$ decrease TKE and transfer energy to potential energy of the mean flow by decreasing the stratification of the interior ocean. Production of TKE is given by downgradient vertical momentum fluxes acting on the shear of the mean flow in the term $-\rho_{0} \overline{\mathbf{u}^{\prime} w^{\prime}} \partial \overline{\mathbf{u}} / \partial z$, that is, transferring energy from the mean flow to TKE by vertical shear instability. We note that the mean flow related to the vertical shear instability might be either the large-scale balanced mean flow or the internal waves. The term $-\nu \rho_{0} \overline{\left(\nabla \mathbf{u}^{\prime}\right)^{2}}$, where $\nu$ denotes dynamical molecular viscosity, decreases TKE and transfers energy to internal energy, that is, heating the ocean (usually at a very small rate). For the derivation of Eq. (1) horizontal isotropy was assumed, which appears a reasonable assumption given the small aspect ratio of the ocean.

Neglecting the flux divergence and the left-hand side, Eq. (1) is often used in the oceanographic context to estimate density mixing rates and associated diffusivities from estimates of dissipation rates (Osborn 1980). To 
close the three term balance, a constant ratio between the first two terms is assumed, that is, a constant flux Richardson number $\mathrm{Ri}_{f}=-g \overline{w^{\prime} \rho} /\left(\rho_{0} \overline{\mathbf{u}^{\prime} w^{\prime}} \partial \overline{\mathbf{u}} / \partial z\right)$. Assuming downgradient eddy fluxes and identical corresponding turbulent diffusivities and viscosities, $\mathrm{Ri}_{f}$ becomes identical to the Richardson number $\mathrm{Ri}=N^{2} /(\partial \overline{\mathbf{u}} / \partial z)^{2}$ with the stability frequency $N^{2}=-g\left(\partial_{z} \bar{\rho}+g \rho_{0} / c_{s}^{2}\right) / \rho_{0}$ and the speed of sound $c_{s}$. From observations and numerical simulation one finds a value for $\mathrm{Ri}_{f}$ close to 0.15 , and thus $g \overline{w^{\prime} \rho^{\prime}} \approx \gamma \nu \rho_{0} \overline{\left(\nabla \mathbf{u}^{\prime}\right)^{2}}$, where $\gamma$ is the "mixing efficiency" $\gamma=\mathrm{Ri}_{f} /\left(1-\mathrm{Ri}_{f}\right) \approx 0.2$ (Gregg et al. 1986; Itsweire et al. 1993), but large deviations of $\gamma$ from this value are possible (Smyth et al. 2001). Estimating the dissipation rate $\overline{\nu\left(\nabla \mathbf{u}^{\prime}\right)^{2}}$ from, for example, microstructure measurements and assuming a downgradient eddy buoyancy flux, the relation allows us to determine the corresponding turbulent diffusivity (this so-called Osborn-Cox relation is used here to fix the parameters $c_{u}$ and $c_{b}$; see below).

To use Eq. (1) in a practical closure, it was proposed by Gaspar et al. (1990) to assume in Eq. (1) downgradient eddy fluxes with essentially identical diffusivity $K$ [besides dimensionless parameters $c_{b}$ and $c_{u}$ of $O(1)$, which are related to each other by $c_{u}=c_{b} \mathrm{Ri}_{\mathrm{R}} / \mathrm{Ri}_{f}$ ] and a parameterization for the dissipation of TKE given by $\nu \overline{\left(\nabla \mathbf{u}^{\prime}\right)^{2}}=c_{\epsilon} \bar{E}_{\mathrm{tke}}^{3 / 2} L^{-1} \equiv \epsilon_{\mathrm{tke}}$ motivated by scaling laws from turbulence theory (e.g., Batchelor 1982), introducing a dissipation length scale $L$ (and another dimensionless parameter $c_{e}$ ), which turns Eq. (1) into

$$
\begin{aligned}
\rho_{0} \frac{d \bar{E}_{\mathrm{tke}}}{d t}= & -\partial_{z}(\text { fluxes })+\rho_{0} c_{u} K\left(\partial_{z} \overline{\mathbf{u}}\right)^{2} \\
& -\rho_{0} c_{b} K N^{2}-\rho_{0} \epsilon_{\mathrm{tke}} .
\end{aligned}
$$

Equation (2) is closed by introducing a mixing length assumption for the diffusivity $K=\bar{E}_{\text {tke }}^{1 / 2} L$, using the same length scale $L$ as for the dissipation, which is determined from another balance equation (in case of higher-order closures) or from simple algebraic relations [in case of the closure by Gaspar et al. (1990)]. One such algebraic relation results from a balance in Eq. (1) between the potential energy gained from raising a particle by $L$ and of that energy by dissipation $\bar{E}_{\mathrm{tke}}^{3 / 2} L^{-1}$, which yields $L=$ $\sqrt{2 \bar{E}_{\mathrm{tke}} / N^{2}}$. Other relations from other possible balances and geometric consideration are combined to the algebraic length scale determination in the parameterization by Gaspar et al. (1990). We follow the treatment of $L$ by Blanke and Delecluse (1993) here.

A closure based on Eq. (2) (or variants of it) is often implemented in ocean circulation models using for $\overline{\mathbf{u}}$ and $N^{2}$ the simulated variables of the model, with reasonable success for simulations in the surface mixed layer (Blanke and Delecluse 1993). In the stratified interior of the model, however, the closure shows a deficit, since the vertical shear of the simulated flow is weak here and the flux of TKE is small, such that almost no local sources of TKE are present. This has the consequence that TKE and the corresponding diffusivity drops to very small values, much smaller than observed. The reason is the missing vertical shear of the internal gravity wave field at high vertical wavenumbers, which remains unresolved by the circulation model, and its missing production of TKE, that is, the missing breaking of internal waves. The problem is often circumvented by applying a minimal threshold of TKE to Eq. (2), from which a minimal threshold of the diffusivity $K=\sqrt{2} E_{\min } / N$ follows. It is clear that such an approach is unphysical, which is resolved in this study using a closure for internal wave energy and dissipation as discussed in the next section.

The forcing for TKE enters Eq. (2) via the vertical boundary condition on the flux divergence term. The surface flux of TKE is related to the energy input by breaking surface gravity waves and is usually parameterized by setting the surface value of TKE (the square of the so-called friction velocity) proportional to the wind stress magnitude (divided by seawater density), which is also done here. The bottom flux of TKE is set to zero here, but might also be related to bottom boundary layers.

\section{b. Internal gravity waves}

Shear instability in the interior of the ocean is assumed to be driven to a large extent by internal waves at high vertical wavenumbers. Those internal waves are in general not resolved by ocean circulation models and thus need parameterization as well. ${ }^{3}$ Here, we use the recently proposed closure for internal gravity wave mixing by Olbers and Eden (2013) that was implemented in the MITgcm model. The closure is based on the radiative transfer balance equation of weakly interacting internal gravity waves in the ocean (Hasselmann 1968). The interaction of the waves can be expressed in principle by a complicated integral describing triad wave-wave interactions for which, however, no comprehensive parameterization exists. In the closure by Olbers and Eden (2013), a few simple assumptions circumvent the specification of the complicated wave-wave interaction. First, the total internal wave energy is split into the energy of upward- and downward-propagating waves by integrating over all horizontal and over all negative or positive

\footnotetext{
${ }^{3}$ Internal waves break typically at vertical wavelengths of $O(10) \mathrm{m}$. To simulate this process in a numerical model, the shear on this spatial scale needs to be resolved, that is, a vertical and horizontal resolution of a couple of meters would be needed.
} 
vertical wavenumbers. It is then assumed that the dissipation of waves acts nearly symmetric with respect to upward- and downward-propagating waves and that the effect of wave-wave interaction is to damp asymmetries in upward- and downward-propagating waves with a time scale $\tau_{v}$ on the order of days.

The dissipation of internal wave energy, that is, the flux into the highest vertical wavenumbers, where internal gravity waves are assumed to break, is parameterized using a quadratic dependency on total wave energy, following an early suggestion by Olbers (1976) (McComas and Müller 1981). This form is supported by Henyey et al. (1986) and is also usually used (in slightly modified form) for estimates of internal wave energy dissipation (Gregg 1989; Polzin et al. 1995; Sun and Kunze 1999). The mean vertical group velocity $c_{0}$ of the upward- or downward-propagating waves is calculated assuming a prescribed spectrum of internal gravity waves, that is, a "GM spectrum" in a form specified by Munk (1981). For time scales longer than $\tau_{v}$, the total energy of internal waves $E_{\text {iw }}$ is governed by

$$
\partial_{t} E_{\mathrm{iw}}=\partial_{z} c_{0} \tau_{v} \partial_{z} c_{0} E_{\mathrm{iw}}+\nabla_{h} \cdot v_{0} \tau_{h} \nabla_{h} v_{0} E_{\mathrm{iw}}-\epsilon_{\mathrm{iw}}
$$

with the parameterization by McComas and Müller (1981) $\epsilon_{\mathrm{iw}}=\mu f E_{\mathrm{iw}}^{2} / c_{\star}^{2}$, with the parameter $\mu=O(1)$, and with $c_{\star}$ related to the bandwidth of the GM spectrum in wavenumber space. The term $v_{0}$ denotes the mean horizontal group velocity of internal waves analogous to $c_{0}$. The vertical symmetrization of internal waves by wave-wave interaction on the time scale $\tau_{v}$ leads to a vertical diffusion of total wave energy $E_{\text {iw }}$. The horizontal anisotropy of the internal wave fields is shown in Olbers and Eden (2013) to be equivalent to zero order to horizontal diffusion of $E_{\text {iw }}$ in Eq. (3), where $\tau_{h}$ is a time scale on the order of days representing the horizontal symmetrization of the wave field. More details on the derivation of the closure for internal wave energy and dissipation can be found in Olbers and Eden (2013). The model for internal waves can be extended with compartments of the low vertical mode near-inertial waves and internal tides as shown in Eden and Olbers (2014) to account for the different lateral propagation characteristics of the low modes, but this extension is not used here. We use an identical parameter as in Olbers and Eden (2013) in the closure Eq. (3).

The forcing of internal waves in the form of energy fluxes enters Eq. (3) as a flux at the surface and bottom via the vertical boundary condition of the flux divergences in Eq. (3) (we use zero fluxes at lateral boundaries). At the surface, this energy flux is thought to be given by wind-generated near-inertial waves radiating down from the surface mixed layer, but other forcing components are also possible (e.g., Olbers and Herterich 1979). Here, we use an estimate of the near-inertial wave flux by Rimac et al. (2013) and no other surface flux. At the bottom, the interaction between the barotropic tides with topographic obstacles generates a flux into the internal wave field. Here, we use an estimate of this flux by Jayne (2009), which was also used in Olbers and Eden (2013). Another source at the bottom could be related to the generation of lee waves by either the mean flow or mesoscale eddies, which was shown by Nikurashin and Ferrari (2011) to be as important as the tidal forcing. To account for this effect, we add the dissipated mesoscale eddy energy as a local forcing to Eq. (3) either at the bottom or the interior, as discussed in the next section.

\section{c. Mesoscale eddies}

A third form of dynamics that is often unresolved in ocean models are mesoscale eddies. Analogous to smallscale turbulence and internal waves, it is useful to describe this kind of turbulent flow also with an energy equation:

$$
\rho_{0} \frac{d \bar{E}_{\mathrm{eke}}}{d t}=-\nabla \cdot(\text { fluxes })+\bar{S}-g \overline{\rho^{\prime} w^{\prime}}-\rho_{0} \epsilon_{\mathrm{eke}},
$$

where $\bar{E}_{\text {eke }}=\overline{\left(u^{\prime 2}+v^{\prime 2}\right)} / 2$ denotes the kinetic energy of mesoscale eddy fluctuations [eddy kinetic energy (EKE)], and $\mathbf{u}^{\prime}$ and $\rho^{\prime}$ denote deviations by mesoscale eddy fluctuations relative to mean velocity $\overline{\mathbf{u}}$ and density $\bar{\rho}$. Since the hydrostatic approximation was applied to derive Eq. (4), the contribution by $\overline{w^{\prime 2}}$ is absent in the kinetic energy. A detailed derivation and discussion of Eq. (4) is provided by many textbooks, for example, Olbers et al. (2012). Besides a flux divergence, three exchange terms show up in Eq. (4): exchange with the mean kinetic $\bar{S}=-\rho_{0} \overline{\mathbf{u}^{\prime} \mathbf{u}^{\prime}} \cdot \nabla_{h} \overline{\mathbf{u}}$ that is given by the eddy momentum flux acting on the lateral shear of the mean flow, exchange with potential energy given by $-g \overline{\rho^{\prime} w^{\prime}}$, and the dissipation of EKE given by $\epsilon_{\text {eke. }}$.

In the context of geophysical fluids, Eq. (4) is often discussed in terms of the so-called Lorenz energy cycle (Lorenz 1955). In this approach, it is convenient to differentiate between available and unavailable potential energy, since for the former the approximate form $P=g^{2} \rho^{2} /\left(2 \rho_{0}^{2} N_{0}^{2}\right)$ can be given, where $N_{0}^{2}(z)$ denotes a stability frequency related to a reference density, usually taken as the horizontally averaged density, and $\rho$ denotes a perturbation from that reference density (Lorenz 1955). Note that an exact definition for available potential energy for the ocean in the presence of compressibility and a nonlinear equation of state was given by Tailleux (2013). For use in parameterizations, 
however, we stick to the approximate one given by Lorenz (1955). Since the approximate available potential energy $P$ is quadratic in the density $\rho$ it becomes possible to define a potential energy related to the mesoscale fluctuations [eddy available potential energy (EPE)] and a potential energy related to the mean flow [mean available potential energy (MPE)]. The former is given by $\bar{P}=g^{2} \overline{\rho^{\prime 2}} /\left(2 \rho_{0}^{2} N^{2}\right)$ and obeys (approximately)

$$
\rho_{0} \frac{d \bar{P}}{d t}=-\nabla \cdot \text { flux }+\delta W_{\mathrm{gm}}+g \overline{\rho^{\prime} w^{\prime}}-\rho_{0} \epsilon_{\mathrm{epe}}
$$

with $\delta W_{\mathrm{gm}}=-\left(g^{2} / \rho_{0}\right) \overline{\mathbf{u}_{h}^{\prime} \rho^{\prime}} \cdot \nabla_{h} \bar{\rho} / N^{2}$. Note that we have replaced the reference stability frequency $N_{0}(z)$ with the full stability frequency $N(x, y, z, t)$ related to the reference plus perturbation density, that is, to the full (Boussinesq) density. A detailed derivation and discussion of Eq. (5) and the underlying approximations are provided by many textbooks, for example, Olbers et al. (2012). Again a flux divergence term and three exchange terms show up in Eq. (5) with interpretation as follows: exchange with mean available potential energy by $\delta W_{\mathrm{gm}}$, exchange with EKE by $g \overline{\rho^{\prime} w^{\prime}}$, and dissipation of $\bar{P}$ by $\epsilon_{\text {epe }}$ related to density mixing by mesoscale eddies. We now add both forms to the total mesoscale eddy energy $E_{\text {eddy }}=\bar{E}_{\text {eke }}+\bar{P}$ to eliminate the vertical eddy density flux and assume a downgradient lateral eddy density flux $\overline{\mathbf{u}_{h}^{\prime} \rho^{\prime}}=-K_{\mathrm{gm}} \nabla_{h} \bar{\rho}$ as in the parameterization by Gent and McWilliams (1990), which yields

$$
\rho_{0} \frac{d E_{\text {eddy }}}{d t}=-\nabla \cdot(\text { fluxes })+\bar{S}+\delta W_{\text {gm }}-\rho_{0} \epsilon_{\text {eddy }},
$$

with $\delta W_{\mathrm{gm}}=\left(g^{2} / \rho_{0}\right) K_{m}\left(\nabla_{h} \bar{\rho}\right)^{2} / N^{2}$ and with $\epsilon_{\mathrm{eddy}}=$ $\epsilon_{\text {eke }}+\epsilon_{\text {epe }}$.

The lateral diffusivity $K_{\mathrm{gm}}$ is identical to the one that has to be specified in the parameterization by Gent and McWilliams (1990). An eddy-induced (or bolus) velocity is given by a streamfunction formed from $K_{\mathrm{gm}}$ times the isopycnal slopes in this parameterization, which adds to the Eulerian mean velocity for tracer advection. However, this definition of the eddy-induced streamfunction is strictly only valid if one assumes vanishing diapycnal eddy buoyancy fluxes or vanishing diapycnal mixing by mesoscale eddies [if one neglects diapycnal rotational eddy flux components that are discussed, for instance, in Eden (2010a)]. This in turn assumes purely viscous dissipation of eddy energy, that is, vanishing dissipation $\epsilon_{\text {epe }}$ and nonlocal terms in Eq. (5) (Tandon and Garrett 1996; Eden and Greatbatch 2008a) and an exact balance between $\delta W_{\mathrm{gm}}$ and $g \overline{\rho^{\prime} w^{\prime}}$. Here, we do not assume vanishing $\epsilon_{\text {epe }}$, but ignore the impact of diapycnal eddy fluxes on the eddy-driven streamfunction, since it should be small.
Estimates of $K_{\mathrm{gm}}$ suggest significant lateral and vertical variations. Closures for $K_{\mathrm{gm}}$ to account for those variations are based, for instance, on Eq. (4) in combination with a mixing length assumption for the skew [Gent-McWilliams (GM)] diffusivity $K_{\text {gm }} \sim \sqrt{\bar{E}_{\text {eddy }}} L$ with a mixing length related to the Rossby radius or Rhines scale (Rhines 1982) and an assumption about the dissipation of eddy energy. Eden and Greatbatch (2008b) use an expression motivated by the dissipation rates in small-scale turbulence as in Eq. (2) and set $\epsilon_{\text {eddy }}=E_{\text {eddy }}^{3 / 2} / L_{d}$ with a dissipation length scale $L_{d}$, while Marshall and Adcroft (2010) use a simple linear decay $\epsilon_{\text {eddy }}=r E_{\text {eddy }}$. Assuming $K_{\text {gm }}=\sqrt{E_{\text {eddy }}} L$ and a local balance between production by baroclinic instability and dissipation in Eq. (6) yields for both closures for the dissipation

$$
E_{\text {eddy }}=c^{2} L^{2} \sigma^{2}, \quad K_{\mathrm{gm}}=c L^{2} \sigma,
$$

with the Eady growth rate $\sigma$ and the parameter $c=\sqrt{L / L_{d}}$ in the first case and $c=\sigma / r$ in the second. The expression for $K_{\mathrm{gm}}$ then becomes analogous to the scalings by Larichev and Held (1995), Held and Larichev (1996), and Visbeck et al. (1997).

Both closures for $\epsilon_{\text {eddy }}$ are, however, problematic since little is known about the dissipation of mesoscale eddy energy. A potentially important mechanism is the lee-wave generation by the mesoscale balanced flow over topography (Bell 1975; Nikurashin and Ferrari 2011), by which the energy of the balanced flow is transferred to the internal gravity wave field. Other possibilities for routes to dissipation of mesoscale eddy energy are the direct leakage of energy of the balanced flow by the Lighthill radiation of gravity waves (Ford et al. 2000), which was estimated by Williams et al. (2008) to be possibly as large as $1.5 \mathrm{TW}$, the direct generation of unbalanced (ageostrophic) instabilities (Molemaker et al. 2005), or simply a direct kinetic energy cascade to smaller scales, which appears to be favored at large Rossby numbers (Capet et al. 2008b; Molemaker et al. 2010). All mechanisms transfer their energy to the internal wave field (or even directly to small-scale turbulence), but they differ in their vertical localization; while lee-wave generation takes place at the bottom, Lighthill radiation might happen anywhere in the interior of the ocean. Since ageostrophic instabilities and a forward kinetic energy cascade are favored by small Richardson numbers (or large Rossby numbers) that are often met in the surface mixed layer, the last mechanism might dissipate mesoscale energy preferable near the surface. To account for the different vertical localizations of $\epsilon_{\text {eddy }}$, we use either the local form of Eden and Greatbatch (2008b) or inject the 
vertically integrated $\epsilon_{\text {eddy }}$ at the bottom to account for lee-wave generation or at the surface to account for the effect of ageostrophic instabilities.

A further key problem for ocean parameterizations is the specification of $\bar{S}$. A simple downgradient closure for $\overline{\mathbf{u}^{\prime} \mathbf{u}^{\prime}}=-A_{h} \nabla_{h} \mathbf{u}_{h}$ yields $\bar{S}=A_{h}\left|\nabla \mathbf{u}_{h}\right|^{2}$, which can be interpreted as dissipation of the mean kinetic energy by harmonic lateral friction. Lateral friction is usually applied as viscous closure in ocean models. However, it is clear that a simple downgradient flux assumption for the eddy momentum flux violates what is known about kinetic energy cascades in geophysical fluids (Rhines 1982). There is evidence from observations and model simulations of an inverse kinetic energy cascade over at least some range of the wavenumber spectrum (Scott and Wang 2005; Schlösser and Eden 2007; Scott and Arbic 2007), although this behavior depends on environmental parameters like the Rossby or Richardson number. A forward cascade of energy appears to dominate for large Rossby numbers, which are met mainly in the surface layers (Capet et al. 2008b; Molemaker et al. 2010), such that ideas about the energy cycle in the ocean based on quasigeostrophic theory (Scott and Arbic 2007) have to be considered with care when applied to the "real" ocean. In geostrophic turbulence, lateral eddy momentum fluxes thus sometimes transfer mean momentum $\mathbf{u}$ in the direction of the lateral gradient of $\mathbf{u}$, that is, "upgradient," implying negative turbulent viscosities (Starr 1968) and thus negative $\bar{S}$ in Eq. (6). We also find such behavior diagnosing $\bar{S}$ in an eddying model simulation by von Storch et al. (2012) below, but also large regions with positive $\bar{S}$.

Nevertheless, it is current practice in ocean models to use lateral (harmonic or biharmonic) friction, sometimes with an anisotropic (but still positive definite) viscosity tensor (Large et al. 2001), which implies downgradient eddy momentum fluxes and strictly positive $\bar{S}$. The reasons to use such unphysical viscous closures are of numerical nature, since current ocean models need large viscous damping for a stable integration. The problem of unphysical viscous closures also applies to eddy-permitting models, for which even less is known about routes to dissipation and plausible closures as for non-eddy-resolving ocean models.

Because of the enstrophy cascade toward smaller scales in quasigeostrophic theory, a physically more plausible assumption is a downgradient eddy potential vorticity flux, which was first proposed by Welander (1973) and which has been discussed by, for example, Marshall (1981), Killworth (1997), Treguier et al. (1997), and more recently by Marshall and Adcroft (2010) and Eden (2010b). However, this idea is hampered by the fact that potential vorticity is not a directly predicted variable in an ocean model based on the primitive equations. As one consequence, care has to be taken in the momentum budget, such that no additional forces are introduced by the parameterization that would otherwise lead to a spurious integral acceleration, since mesoscale eddy momentum fluxes only redistribute but do not create momentum (Bretherton 1966). We do not attempt to implement potential vorticity diffusion in our model at this stage but keep the standard approach of harmonic friction for simplicity and to demonstrate that an energetically consistent model is still possible. The dissipated mean kinetic energy by $\bar{S}$ is injected together with $\delta W_{\text {gm }}$ into the eddy energy Eq. (6).

Since the closure of $\bar{S}$ represents a possible important bias in our model simulation, we compare the simulated fields of $\bar{S}$ and $\delta W_{\mathrm{gm}}$ below with an eddy-permitting model simulation by von Storch et al. (2012). Although also certainly biased by unphysical closures and other issues, such an eddying model simulation might at least give a zero-order impression of the fields of $\bar{S}$ and $\delta W_{\mathrm{gm}}$. We use the comparison as a rough validation of the local and global magnitude of energy transferred from mean energy to EKE, which is then further transported laterally and vertically by the EKE and the internal wave closure to be used for density mixing.

\section{d. Connecting parameterizations in a consistent way}

Since all parameterizations listed above are based on energetic considerations, it is possible now to link all forms of parameterized dynamics in an energetically consistent form for use in an ocean general circulation model:

$$
\begin{aligned}
\rho_{0} \frac{d E_{\mathrm{gcm}}}{d t}= & -\nabla \cdot(\text { fluxes })-\bar{S}-\delta W_{\mathrm{gm}} \\
& -\rho_{0} c_{u} K\left(\partial_{z} \overline{\mathbf{u}}\right)^{2}+\rho_{0} c_{b} K N^{2}, \\
\rho_{0} \frac{d E_{\mathrm{eddy}}}{d t}= & -\nabla \cdot(\text { fluxes })+\bar{S}+\delta W_{\mathrm{gm}}-\rho_{0} \epsilon_{\mathrm{eddy}}, \\
\rho_{0} \frac{d E_{\mathrm{iw}}}{d t}= & -\nabla \cdot(\text { fluxes })-\rho_{0} \epsilon_{\mathrm{iw}}+\rho_{0} \epsilon_{\mathrm{eddy}}, \text { and } \\
\rho_{0} \frac{d \bar{E}_{\mathrm{tke}}}{d t}= & -\nabla \cdot(\text { fluxes })+\rho_{0} \epsilon_{\mathrm{iw}}+\rho_{0} c_{u} K\left(\partial_{z} \overline{\mathbf{u}}\right)^{2} \\
& -\rho_{0} c_{b} K N^{2}-\rho_{0} \epsilon_{\mathrm{tke}} \cdot
\end{aligned}
$$

The term $E_{\mathrm{gcm}}$ contains the total energy of the mean variables of the ocean model. In the fully compressible equations, total energy is composed of kinetic, potential, and internal energy (Olbers et al. 2012). In Boussinesq approximation, which we apply in our model, equivalent components of the total energy can be defined as 
detailed in a companion study (Eden 2014, manuscript submitted to J. Phys. Oceanogr.) to allow for a closed and consistent energy cycle of ocean models. Instead of using internal energy and potential energy, it is, however, more convenient to define potential and dynamic enthalpy to approximately differentiate between reversible and irreversible energy exchanges (McDougall 2003; Young 2010; Nycander 2011), both for the compressible and Boussinesq equations. Potential enthalpy then replaces temperature as the thermodynamic state variable and is also called conservative temperature. Effects of compressibility and the nonlinear equation of state on the energy exchanges between the mean and parameterized forms of energy that raise additional energy exchange terms will be discussed in a later study and are simply ignored here (see also section 4 and the discussion).

Sinks of $E_{\mathrm{gcm}}$ depend on the specific closures. For the previously discussed closures, they are given by the parameterization by Gent and McWilliams (1990) $\left(\delta W_{\mathrm{gm}}\right)$, lateral friction $\left[\bar{S}=\rho_{0} A_{h}\left(\nabla_{h} \overline{\mathbf{u}}_{h}\right)^{2}\right.$ with lateral viscosity $\left.A_{h}=5 \times 10^{4} \mathrm{~m}^{2} \mathrm{~s}^{-1}\right]$, vertical friction $\left[\rho_{0} c_{u} K\left(\partial_{z} \overline{\mathbf{u}}\right)^{2}\right]$, and vertical (or diapycnal) mixing $\left(\rho_{0} c_{b} K N^{2}\right)$. To obtain a consistent energy cycle of the ocean model without spurious sources and sinks, these energy fluxes are transferred to the parameterized forms of energy.

The previously discussed energy transfers all show up in the energy cycle Eqs. (8) to (11). To obtain a closed energy cycle, two important interactions are added: dissipation of internal wave energy is transferred to small-scale turbulence and the dissipation of mesoscale eddy energy is transferred to the internal wave energy. It is clear that for the latter, other choices can be made instead. For instance, dissipation of $E_{\text {eddy }}$ could be transferred at least in parts directly to the small-scale turbulence in a bottom or surface boundary layer, for reasons explained above. We have in particular not accounted for a bottom boundary layer using some kind of bottom friction, where $E_{\text {eddy }}$ is transferred directly to $E_{\mathrm{tke}}$, since we have not much information about the importance of such processes. On the other hand, bottom friction could be easily implemented in the concept. Alternative choices for the dissipation of $E_{\text {eddy }}$ are explored here only in an ad hoc and simple way in a series of sensitivity experiments, but it is clear that those ways are premature and need improvement. Here, we aim to demonstrate the possibility for an energetically consistent ocean model using simple but reasonable closures. Today's ocean models are not consistent in this respect.

Nonlocal transport terms are present in Eqs. (9) to (11), which also need some discussion. In Eq. (11) horizontal homogeneity is assumed such that the nonlocal flux on the right-hand side becomes vertical. Since it is predominantly active in the surface mixed layer, and since advection by the mean flow is also neglected in Eq. (11), it turns into a local balance in the interior of the ocean, that is, the Osborn-Cox relation.

In the internal wave balance Eq. (10), Olbers and Eden (2013) also neglected the mean advection in the radiative energy balance, although it could be easily incorporated as well. Similar to Eq. (11), preliminary tests suggest that the impact of mean advection is very small, since time scales of generation and dissipation are smaller than the advective time scale. The nonlocal transport on the right-hand side of Eqs. (10) or (3) is, however, of key importance. It contains the effect of wave-wave interaction represented by a vertical and a horizontal component. The vertical component describes the symmetrization of the wave field with respect to vertical wavenumber and determines to a large extent the vertical profile of energy dissipation. It also connects the surface and bottom external fluxes by tides and winds to the internal wave field. Since the horizontal flux depends on wavenumber in a more complex way as implemented in Eq. (3), representing a zero-order closure for the effect, Eden and Olbers (2014) discussed an extension of the closure to account for low-mode tidal and near-inertial wave components. Preliminary tests suggest that the impact of an improved representation of low modes is small compared to the simpler version given by Eq. (3). The effect will be discussed in detail in a later study.

In Eq. (9) we incorporate the advection by the mean flow since tests show that it has some effect in strong boundary currents. As in Eden and Greatbatch (2008b), we use for the nonlocal transport term on the right-hand side of Eq. (9) simply lateral and vertical diffusion (using $K_{\mathrm{gm}}$ and $K_{\mathrm{gm}} f^{2} / N^{2}$ as diffusivities, respectively) as a zero-order closure for lateral and vertical energy radiation. A better closure might be a constant westward advection as used in Marshall and Adcroft (2010), but the effect of the nonlocal terms will still be minor assuming that production and decay time scales are smaller than propagation time scales. In fact, a local version of Eq. (9) as discussed in Eden et al. (2009) produces rather similar results in a coarse resolution model. However, we do see a strong sensitivity of the model results on the localization of the dissipation term in Eq. (9), as discussed below.

Adding all forms of energy together yields indeed a consistent energy cycle without spurious sources or sinks of energy:

$$
\rho_{0} \frac{d}{d t}\left(E_{\mathrm{gcm}}+E_{\mathrm{eddy}}+E_{\mathrm{iw}}+\bar{E}_{\mathrm{tke}}\right)=-\nabla \cdot(\text { fluxes })-\rho_{0} \epsilon_{\mathrm{tke}} .
$$


This can be seen by integrating Eq. (12) over a closed domain since energy is then added only by external fluxes (forcing from the atmosphere and the tides) and removed by conversion to internal energy, while all nonlocal flux terms will vanish. This internal heating rate $\epsilon_{\mathrm{tke}}$ could be used in the (conservative) temperature equation-which is in fact part of $E_{\mathrm{gcm}}$-but the rates are smaller compared to other already neglected sources (e.g., McDougall 2003) and should thus be ignored.

\section{Results}

\section{a. List of experiments}

We discuss the following experiments:

- STANDARD, in which mesoscale eddy energy Eq. (9) and internal wave energy Eq. (10) are not considered and with the small-scale closure Eq. (11) in the form

$$
\begin{aligned}
\rho_{0} \frac{d \bar{E}_{\mathrm{tke}}}{d t}= & -\nabla \cdot(\text { fluxes })+c_{u} \rho_{0} K\left(\partial_{z} \overline{\mathbf{u}}\right)^{2} \\
& -\rho_{0} c_{b} K N^{2}-\rho_{0} \epsilon_{\mathrm{tke}} .
\end{aligned}
$$

The skew (GM) diffusivity is set to $K_{\mathrm{gm}}=1000 \mathrm{~m}^{2} \mathrm{~s}^{-1}$, and $\bar{E}_{\mathrm{tke}}$ is set to a minimal threshold, $\bar{E}_{\mathrm{tke}} \rightarrow$ $\max \left(10^{-6} \mathrm{~m}^{2} \mathrm{~s}^{-2}, \bar{E}_{\mathrm{tke}}\right)$, to allow for nonvanishing diffusivities $K$ in the interior. This configuration corresponds to a state-of-the-art (standard) ocean model. In the energy cycle, the mesoscale energy production terms $\bar{S}+\delta W_{\mathrm{gm}}$ are lost, and the dissipation of mesoscale eddy energy $\epsilon_{\text {eddy }}$ is also lost, while energy is spuriously introduced in Eq. (13) by the threshold for $\bar{E}_{\text {tke }}$.

- WAVE is the same as STANDARD but including the closure for internal wave dissipation

$$
\rho_{0} \frac{d E_{\mathrm{iw}}}{d t}=-\nabla \cdot(\text { fluxes })-\rho_{0} \epsilon_{\mathrm{iw}}
$$

and adding in Eq. (13) the internal wave dissipation $\epsilon_{\mathrm{iw}}$ :

$$
\begin{aligned}
\rho_{0} \frac{d \bar{E}_{\mathrm{tke}}}{d t}= & -\nabla \cdot(\text { fluxes })+c_{u} \rho_{0} K\left(\partial_{z} \overline{\mathbf{u}}\right)^{2} \\
& -\rho_{0} c_{b} K N^{2}+\rho_{0} \epsilon_{\mathrm{iw}}-\rho_{0} \epsilon_{\mathrm{tke}}
\end{aligned}
$$

Bottom and surface fluxes of $E_{\text {iw }}$ given by tides and winds, respectively, are boundary conditions for the flux divergence in Eq. (14). There is no minimal threshold for $\bar{E}_{\text {tke }}$ and thus no spurious energy production in Eq. (15). In the energy cycle, however, the mesoscale energy production terms $\bar{S}+\delta W_{\mathrm{gm}}$ and the dissipation of mesoscale eddy energy $\epsilon_{\text {eddy }}$ are still lost.
- GM-INT is the same as WAVE but including a local version of Eq. (9), in which the eddy energy production $\bar{S}$ is neglected,

$$
0=\delta W_{\mathrm{gm}}-\rho_{0} \epsilon_{\mathrm{eddy}},
$$

and adding in Eq. (14) the dissipation of mesoscale eddy energy $\epsilon_{\text {eddy }}$,

$$
\rho_{0} \frac{d E_{\mathrm{iw}}}{d t}=-\nabla \cdot(\text { fluxes })-\rho_{0} \epsilon_{\mathrm{iw}}+\rho_{0} \epsilon_{\mathrm{eddy}} .
$$

The skew (GM) diffusivity is still set to $K_{\mathrm{gm}}=$ $1000 \mathrm{~m}^{2} \mathrm{~s}^{-1}$. The dissipation of mesoscale eddy energy is assumed to take place locally, corresponding to interior loss of balance. In the energy cycle, the mesoscale energy production term $\bar{S}$ is still lost.

- CONSIST-INT features a fully consistent energy cycle, that is, as in Eqs. (8) to (11). The dissipation of mesoscale eddy energy is parameterized as $\epsilon_{\text {eddy }}=E_{\text {eddy }}^{3 / 2} / L$, the skew (GM) diffusivity as $K_{\mathrm{gm}}=E_{\text {eddy }}^{1 / 2} L$, and the eddy length scale as $L=\min \left(L_{r}, L_{\mathrm{Rhi}}\right)$, where $L_{r}=$ $\int_{-h}^{0} N d z /(\pi|f|)$ denotes the local Rossby radius ${ }^{4}$ and $L_{\text {Rhi }}$ denotes the Rhines scale. The energy transfer $\epsilon_{\text {eddy }}$ is injected locally to the internal wave field, which corresponds to the local loss of balance of mesoscale eddies everywhere in the interior of the ocean.

- CONSIST-BOT is the same as CONSIST-INT, but the mesoscale eddy energy is injected entirely at the bottom into the internal wave field, that is, Eq. (10) is replaced by

$$
\begin{aligned}
\rho_{0} \frac{d E_{\mathrm{iw}}}{d t}= & -\nabla \cdot(\text { fluxes })-\rho_{0} \epsilon_{\mathrm{iw}} \\
& +\delta(z+h) \int_{-h}^{0} d z \rho_{0} \epsilon_{\text {eddy }} .
\end{aligned}
$$

This treatment of $\epsilon_{\text {eddy }}$ corresponds to the dissipation of mesoscale eddies by lee-wave generation by flow over topographic obstacles.

- CONSIST-SURF is the same as CONSIST-INT, but the mesoscale eddy energy is partly injected at the bottom into the internal wave field and partly at the surface into the small-scale turbulence, that is, Eq. (10) is replaced by

$$
\begin{aligned}
\rho_{0} \frac{d E_{\mathrm{iw}}}{d t}= & -\nabla \cdot(\text { fluxes })-\rho_{0} \epsilon_{\mathrm{iw}} \\
& +0.2 \delta(z+h) \int_{-h}^{0} d z \rho_{0} \epsilon_{\text {eddy }},
\end{aligned}
$$

and Eq. (11) is replaced by

\footnotetext{
${ }^{4}$ The midlatitude Rossby radius is replaced by the equatorial Rossby radius close the equator to prevent a singularity as in Eden et al. (2009).
} 
a)

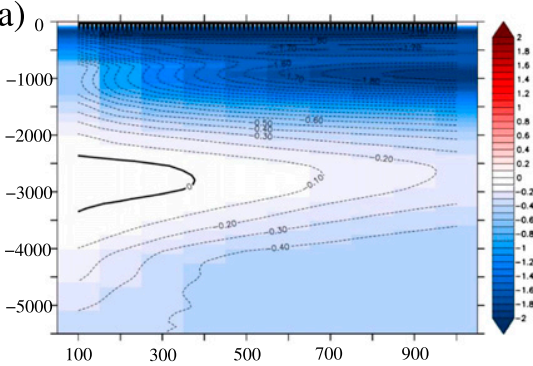

d)

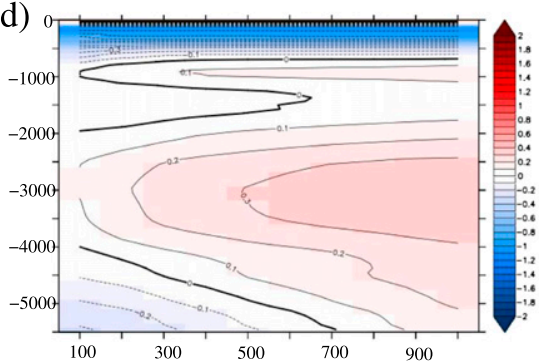

b)

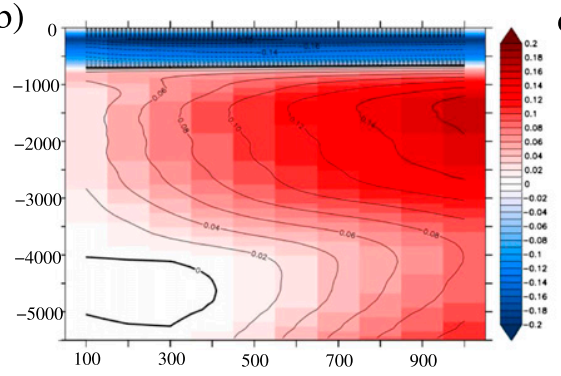

e)

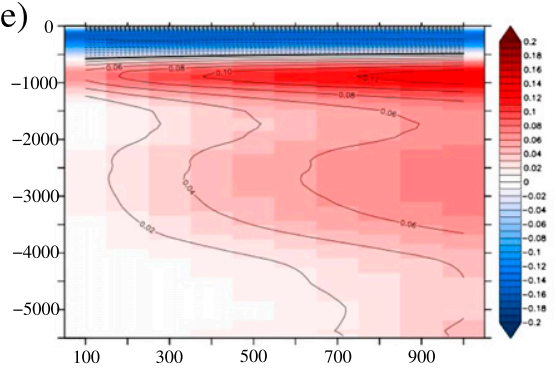

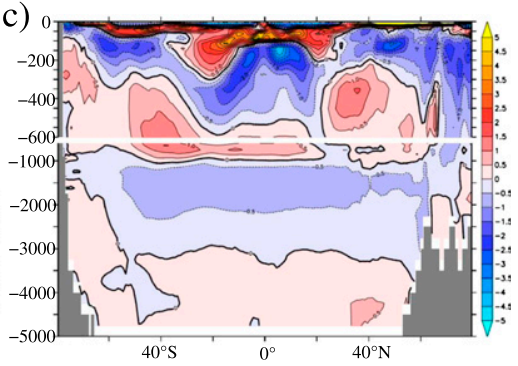

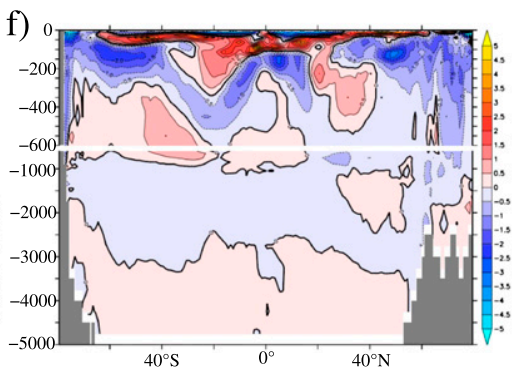

FIG. 2. Time series of horizontally averaged (a),(d) temperature $\left({ }^{\circ} \mathrm{C}\right)$ and (b),(e) salinity $\left(\mathrm{g} \mathrm{kg}^{-1}\right)$ in experiment (top) STANDARD and in (bottom) CONSIST-SURF as a function of depth $(\mathrm{m})$ and time (yr). Shown are 100-yr averages relative to the initial condition. Contour interval is $0.1 \mathrm{k}$ for temperature and $0.02 \mathrm{~g} \mathrm{~kg}^{-1}$ for salinity. Also shown is the difference of the zonally averaged stability frequency $N$ to the climatology of Johnson et al. (2009) $\left(10^{3} \mathrm{~s}^{-1}\right)$ in (c) STANDARD and (f) CONSIST-SURF at the end of the simulation. Contour interval is $0.5 \times 10^{3} \mathrm{~s}^{-1}$.

$$
\begin{aligned}
\rho_{0} \frac{d \bar{E}_{\mathrm{tke}}}{d t}= & -\nabla \cdot(\text { fluxes })+c_{u} \rho_{0} K\left(\partial_{z} \overline{\mathbf{u}}\right)^{2}+g c_{b} K \partial_{z} \bar{\rho} \\
& +\rho_{0} \epsilon_{\mathrm{iw}}-\rho_{0} \epsilon_{\mathrm{tke}}+0.8 \delta(z) \int_{-h}^{0} d z \rho_{0} \epsilon_{\mathrm{eddy}}
\end{aligned}
$$

This treatment of $\epsilon_{\text {eddy }}$ corresponds to the combined effect of lee-wave generation at the bottom and dissipation of mesoscale energy by ageostrophic instability in the mixed layer of the ocean. The bulk of the energy, that is, $80 \%$, is injected in the mixed layer.

Only the model versions CONSIST-INT, CONSISTBOT, and CONSIST-SURF are energetically consistent, that is, no form of energy is lost (expect for numerical biases and complications due to the nonlinear equation of state), and a comprehensive account of the energy cycle in the ocean becomes possible. The other model versions are energetically inconsistent since they contain spurious sources or sinks of energy.

All model versions are integrated for $1000 \mathrm{yr}$, and we made no efforts to tune the model toward a better agreement with observations. Horizontally averaged time series of temperature and salinity (Fig. 2) show a large drift from the initial conditions-which are taken from observations-during the first few hundred years of integration and still considerable drift at the end of the integrations, but we made no attempt to extend the integrations to fully establish a diffusive equilibrium. While the temperature drift in STANDARD is largely reduced in the consistent experiment CONSIST-SURF, there is less reduction of the salinity drift. The temperature drift in STANDARD is comparable, but the temperature drift in CONSIST-SURF is somewhat reduced compared to the drift of other global ocean models (Griffies et al. 2009). The remaining biases in salinity are comparable to the ones shown in Griffies et al. (2009) in both STANDARD and CONSIST-SURF. Figure 2 also shows the difference of the zonally averaged stability frequency $N$ with respect to the climatology of Johnson et al. (2009) at the end of the simulation. Biases in $N$, in particular in the main thermocline from 1000- to 200-m depth are largely reduced in CONSIST-SURF compared to STANDARD, although still present. The remaining biases in $N$ are mostly related to biases in the salinity gradients at high latitudes and to temperature gradients in the subtropics and tropics.

The remaining biases in temperature, salinity, and stratification in the consistent simulations are related to several issues. One is the missing the Arctic Ocean and missing water mass conversion regions. The missing cold water masses and related missing cold Denmark and Faroe Strait Overflow leads to warm biases in the deep Atlantic Ocean, which is a well-known model bias (even 

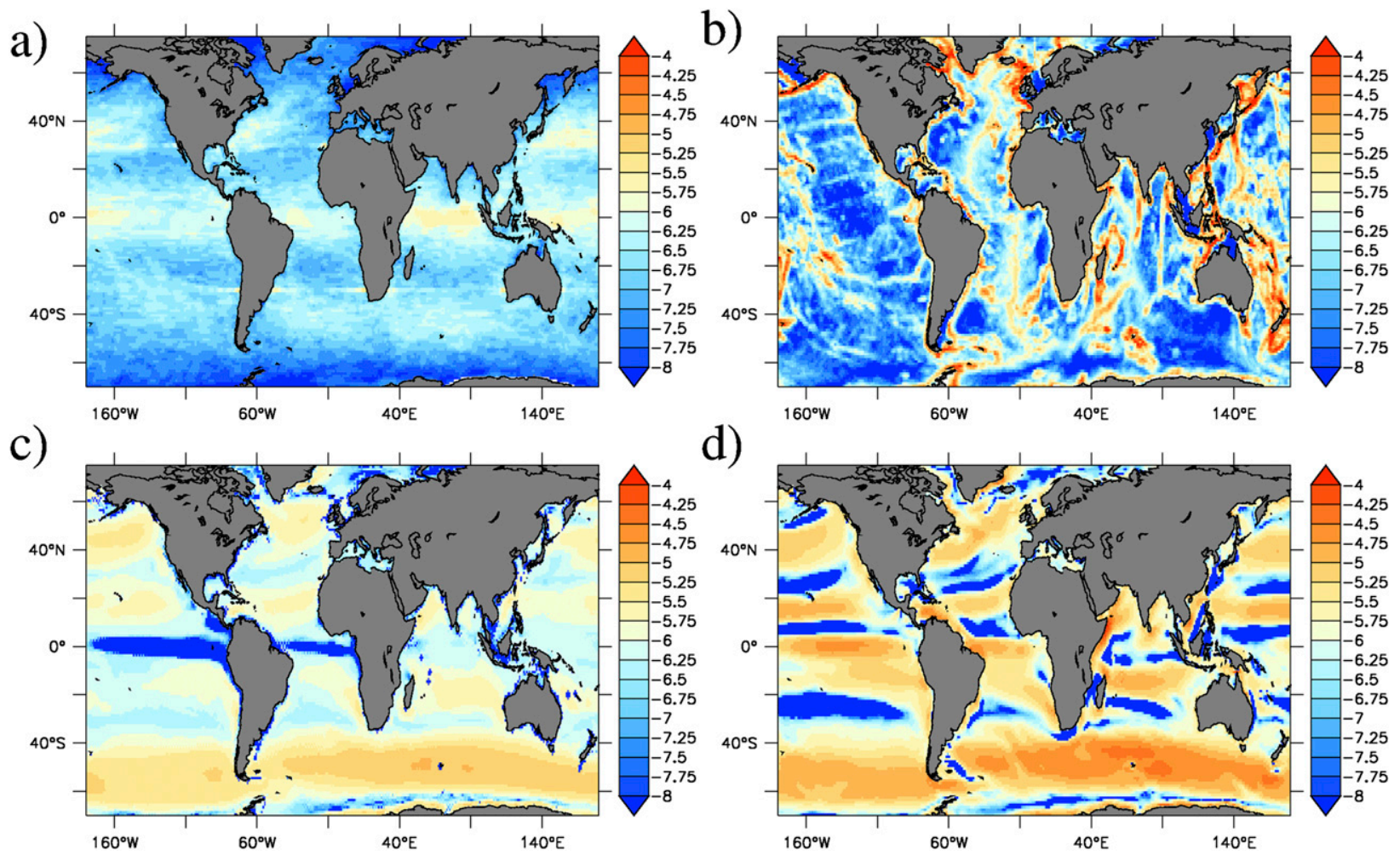

FIG. 3. $\log _{10}$-scaled energy forcing $\left(\mathrm{m}^{3} \mathrm{~s}^{-3}\right)$ in CONSIST-INT. (a) Surface energy flux $F_{s}$ into internal waves. (b) Bottom flux into internal waves. (c) Surface forcing of small-scale turbulence. (d) Surface wind forcing $\boldsymbol{\tau} \cdot \overline{\boldsymbol{u}}_{h}(z=0)$. Values smaller than $10^{-8} \mathrm{~m}^{3} \mathrm{~s}^{-3}$ including negative values are shown in dark blue.

when including the Arctic). A further problematic region is the water mass formation near the Antarctic continent that involves sea ice formation (we do not include a sea ice model). We do not expect such model biases to change when improving mixing parameterizations.

\section{b. Forcing functions and total dissipation}

Figure 3 shows the energy forcing functions of CONSIST-INT originating either from the atmosphere or the tidal system. The surface forcing of internal waves by near-inertial waves radiating from the surface mixed layer is shown in Fig. 3a and amounts globally to $0.14 \mathrm{TW}$. The bottom flux into the internal waves by the tidal forcing, shown in Fig. 3b, is much larger and amounts to 1.8 TW. The surface flux of small-scale turbulence is shown in Fig. 3c, diagnosed from the diffusive flux of $E_{\mathrm{tke}}$ in the first grid box of the model. ${ }^{5}$ Its globally integrated value is $0.27 \mathrm{TW}$ (see Table 1 ). The surface work

\footnotetext{
${ }^{5}$ In the model by Gaspar et al. (1990), as implemented in MITgcm, a Dirichlet surface boundary condition for $E_{\mathrm{tke}}$ is used. The flux diagnosed from the Dirichlet boundary condition becomes negative over the tropical Pacific and Atlantic Oceans, pointing toward the need for improvement of the surface flux boundary condition in the closure by Gaspar et al. (1990).
}

by the winds on the mean flow is shown in Fig. 3d and amounts to $1.83 \mathrm{TW}$. The differences in the forcing among the experiments are mainly due to variations in the surface flux of TKE, as shown in Table 1. It almost vanishes in the global integral in CONSIST-SURF. Since we add part of the dissipation of mesoscale eddy energy to the mixed layer in this experiment, a flux to the atmosphere in regions with strong mesoscale eddy dissipation such as in boundary currents and tropical current system develops. It is clear that this loss of TKE into the atmosphere is an artifact of the Dirichlet boundary condition for TKE, but no attempt to implement a flux boundary condition instead was made in this study.

All the energy input by the external forcing is in the consistent experiments transferred internally within the energy cycle of the model and is finally dissipated to internal energy (heat). The sum of the forcing amounts globally to $4.0 \mathrm{TW}$ in CONSIST-INT and only slightly smaller in CONSIST-BOT. We have also estimated independently the total dissipation of small-scale turbulence given by $\epsilon_{\mathrm{tke}}=c_{\epsilon} \bar{E}_{\mathrm{tke}}^{3 / 2} / L$ as $4.02 \mathrm{TW}$ in CONSIST-INT, indicating that there is still a small spurious source of energy of a few percent in the model. Such imbalances are also present in CONSIST-SURF and CONSISTBOT (see Table 1) and further discussed in section 4. 
TABLE 1. Energy transfers and fluxes $\left(10^{12} \mathrm{~W}\right)$. All values are direct diagnostics; the small imbalances in the consistent model versions between total forcing and $\epsilon_{\mathrm{tke}}$ and the sum of internal wave forcing and $\delta W_{\mathrm{gm}}+\bar{S}$ and $\epsilon_{\mathrm{iw}}$ are due to numerical errors and the nonlinear equation of state.

\begin{tabular}{lcccccc}
\hline \hline & STANDARD & WAVE & GM-INT & CONSIST-INT & CONSIST-BOT & CONSIST-SURF \\
\hline Internal wave forcing & - & 1.91 & 1.91 & 1.91 & 1.91 & 1.91 \\
$\rho_{0} \int \overline{\mathbf{u}} \cdot \boldsymbol{\tau} d A$ & 1.81 & 1.82 & 1.76 & 1.83 & 1.83 & 1.86 \\
$\rho_{0} \int F_{\mathrm{tke}} d A$ & 0.20 & 0.21 & 0.22 & 0.27 & 0.26 & 0.01 \\
Tot forcing & 2.01 & 3.93 & 3.89 & 4.00 & 4.00 & 3.75 \\
$\rho_{0} \int \epsilon_{\mathrm{tke}} d V$ & 2.42 & 3.41 & 3.72 & 4.02 & 3.93 & 3.81 \\
$\rho_{0} \int \epsilon_{\mathrm{iw}} d V$ & - & 2.01 & 2.65 & 2.86 & 2.84 & 2.03 \\
$\int_{-h}^{0} \delta W_{\mathrm{gm}} / \rho_{0} d z$ & - & - & - & 0.58 & 0.58 & 0.56 \\
$\int_{-h}^{0} \bar{S} / \rho_{0} d z$ & - & - & - & 0.46 & 0.47 & 0.45 \\
\hline
\end{tabular}

\section{c. Internal energy exchanges}

The internal exchanges due to the interactions between the different dynamical regimes in the simulations are on the same order of magnitude as the forcing functions. Figure 4a shows the vertically integrated energy flux due to baroclinic instability $\int_{-h}^{0} \delta W_{\mathrm{gm}} / \rho_{0} d z$ from the mean energy to the mesoscale eddy energy by the Gent and McWilliams (1990) parameterization. It amounts in the global integral to $0.58 \mathrm{TW}$ in CONSIST-INT, which is very similar in the other experiments. The vertically integrated flux to the eddy field due to lateral friction $\int_{-h}^{0} \bar{S} / \rho_{0} d z$ is nearly of the same magnitude (Fig. $4 \mathrm{~b}$ ) and amounts to $0.46 \mathrm{TW}$, again similar in the other experiments.

Since there are no other sources or surface fluxes for mesoscale eddy energy in Eq. (6), both add in the global integral to the dissipation of mesoscale eddy energy $\epsilon_{\text {eddy. }}$ This dissipated energy in turn forces the internal wave energy in CONSIST-INT and CONSIST-BOT, in addition to the external fluxes shown in Figs. $3 a$ and $3 b$. In CONSIST-SURF, only $20 \%$ of the dissipated mesoscale energy is transferred to the internal wave energy, while $80 \%$ is transferred to TKE in the mixed layer. The dissipation of internal wave energy is shown in Fig. 4c and amounts to $2.86 \mathrm{TW}$ in CONSIST-INT and similarly in CONSIST-BOT, while in CONSIST-SURF the dissipation only amounts to $2.03 \mathrm{TW}$. Since the surface flux of TKE is small and consumed predominantly in the surface mixed layer, and since vertical shear instability of the mean flow in Eq. (2) is also small in the interior of the ocean, the dissipated internal wave energy is the only source for mixing in the interior. Our TKE closure generates in the interior a flux Richardson number of 0.15 , such that we find an energy transfer of only about
0.43 TW (0.31 TW in CONSIST-SURF) into the mean potential energy due to breaking internal waves $\left[c_{b} K N^{2}\right.$ in Eq. (8)] that contributes to drive the large-scale circulation (e.g., the meridional overturning circulation). A fraction of the flux into the internal wave field comes from the dissipation of mesoscale eddies, while the dominant part comes from tidal forcing (plus a minor part from near-inertial waves). This fraction (about 10\% in CONSIST-SURF to $40 \%$ in CONSIST-INT) depends on the mechanism and thus vertical localization of eddy dissipation. The energy flux due to breaking waves is much less than the direct energy forcing of the largescale circulation by the wind via $\overline{\mathbf{u}} \cdot \tau$.

\section{d. Energy reservoirs}

Energy reservoirs are listed in Table 2 . The mean kinetic energy is on the order of $0.2 \times 10^{18} \mathrm{~J}$ and is slightly larger in the consistent model versions. In Table 2, we also show the mean gravitational potential energy $g\left(\rho-\rho_{0}\right) z$. There is a rough correspondence between the amount of energy available for mixing and the potential energy in the different experiments: the less $\epsilon_{\mathrm{tke}}$ is available, the deeper the potential energy drops. The potential energy of the completely mixed ocean is $-478.1 \times 10^{21} \mathrm{~J}$, that is, only slightly larger than in all model versions. Note that from the difference of potential energy between a completely mixed ocean and the actual state-which is on the order of $1-7 \times 10^{21} \mathrm{~J}$ and therefore still an order of magnitude larger than the kinetic energy_only a small fraction is available for setting the ocean into motion, while the rest can only be assessed by diabatic processes. However, we do not analyze the available potential energy further here. 
a)

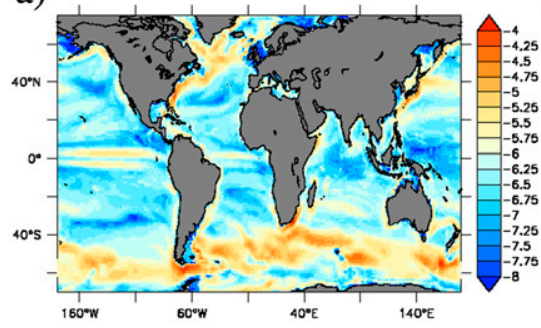

b)

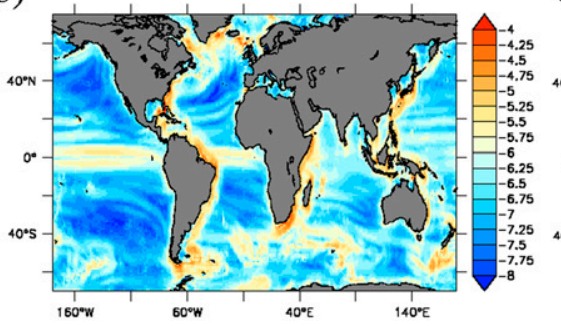

c)

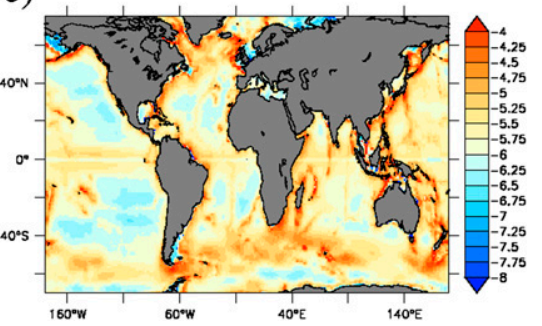

FIG. 4. $\log _{10}$-scaled internal energy transfers $\left(\mathrm{m}^{3} \mathrm{~s}^{-3}\right)$ in CONSIST-INT. (a) Energy transfer due to baroclinic instability $\int_{-h}^{0} \delta W_{\mathrm{gm}} / \rho_{0} d z$. (b) Energy transfer due to barotropic instability $\int_{-h}^{0} \bar{S} / \rho_{0} d z$. (c) Dissipation of internal wave energy $\int_{-h}^{0} \epsilon_{\mathrm{iw}} d z$.

Although not directly related to the other mechanical energy forms, we also discuss the heat content of the models. Variations of the potential enthalpy (heat) among the experiments are on the order of $5 \times$ $10^{24} \mathrm{~J}$, that is, again three orders of magnitude larger than the variations in potential energy. The smallest heat content can be found in the model with the smallest $\epsilon_{\mathrm{tke}}$. The total potential enthalpy is again three orders of magnitude larger and amounts to more than $10^{27} \mathrm{~J}$.

The reservoir of $E_{\mathrm{tke}}$ is on the order of $10^{15} \mathrm{~J}$, that is, up to three orders of magnitude smaller than mean kinetic, internal wave, or mesoscale eddy energy. There is about twice as much internal wave energy as mean kinetic energy, but note that $E_{\text {iw }}$ contains both the kinetic and potential energy of internal waves. The same holds for mesoscale eddy energy, which is again twice as large as internal wave energy. Figure 5a shows the horizontal distribution of $E_{\text {eddy }}$ in CONSISTSURF, which is very similar in the other consistent model versions. Eddy energy is large in the western boundary currents and Southern Ocean. The resulting diffusivity $K_{\mathrm{gm}}$ for the parameterization by Gent and McWilliams (1990) is shown in Fig. 5c. The magnitude and lateral distribution of $K_{\mathrm{gm}}$ agrees with the model simulation in Eden et al. (2009) and, for example, estimates of $K_{\mathrm{gm}}$ based on linear stability analysis by Vollmer and Eden (2013). As $E_{\text {eddy }}$, it is large in the western boundary currents and the Southern Ocean and also shows increased values in the subtropical westward return currents.

\section{e. Comparison of energy fluxes and reservoirs with observational estimates and eddy-permitting model results}

The wind forcing of the mean kinetic energy varies little over the different experiments (Table 1) and agrees well with the estimate of $1.85 \mathrm{TW}$ by von Storch et al. (2012) using a realistic eddying global ocean model. The value is larger than those reported from observations (Wunsch 1998; Scott and Xu 2009) since the model-based estimates also include wind work at the ageostrophic flow [which is mostly dissipated within the surface mixed layer (Roquet et al. 2011)]. Von Storch et al. (2012) also find close agreement of the wind work to a different eddying global ocean model (von Storch et al. 2007) for which the wind work on the geostrophic flow was in turn in close agreement with the observational estimates (although at the larger end of the observational spread). We thus conclude that the wind work on the mean circulation in our experiments is in agreement with observational estimates.

On the other hand, the surface forcing of mesoscale eddies has been excluded in our study. The wind forcing of the time-varying surface flow is found to be $2.19 \mathrm{TW}$ in von Storch et al. (2012). Part of that forcing due to the fluctuating winds drives near-inertial waves since highfrequency wind forcing was used in the eddying model

TABLE 2. Globally integrated energies. KE denotes mean kinetic energy, $\rho_{0}\left|\mathbf{u}_{h}\right|^{2} ;$ PE denotes mean gravitational potential energy $g\left(\rho-\rho_{0}\right) z$. HEAT denotes potential enthalpy $c_{p}^{0} \Theta$ where $\Theta$ is the temperature variable of the model and $c_{p}^{0}$ is a fixed heat capacity. From HEAT a constant value of $1.443 \times 10^{27} \mathrm{~J}$ was subtracted.

\begin{tabular}{|c|c|c|c|c|c|c|}
\hline & STANDARD & WAVE & GM-INT & CONSIST-INT & CONSIST-BOT & CONSIST-SURF \\
\hline $\operatorname{KE}\left(10^{18} \mathrm{~J}\right)$ & 0.23 & 0.24 & 0.22 & 0.28 & 0.28 & 0.28 \\
\hline $\operatorname{PE}\left(10^{21} \mathrm{~J}\right)$ & -485.1 & -482.0 & -479.6 & -480.2 & -479.9 & -480.8 \\
\hline HEAT $\left(10^{24} \mathrm{~J}\right)$ & 14.75 & 17.46 & 18.86 & 19.89 & 19.99 & 19.74 \\
\hline$E_{\mathrm{tke}}\left(10^{15} \mathrm{~J}\right)$ & 2.9 & 4.7 & 6.6 & 5.8 & 6.5 & 5.2 \\
\hline$E_{\mathrm{iw}}\left(10^{18} \mathrm{~J}\right)$ & - & 0.38 & 0.50 & 0.54 & 0.59 & 0.4 \\
\hline$E_{\text {eddy }}\left(10^{18} \mathrm{~J}\right)$ & - & - & - & 1.14 & 1.14 & 1.11 \\
\hline
\end{tabular}


a)

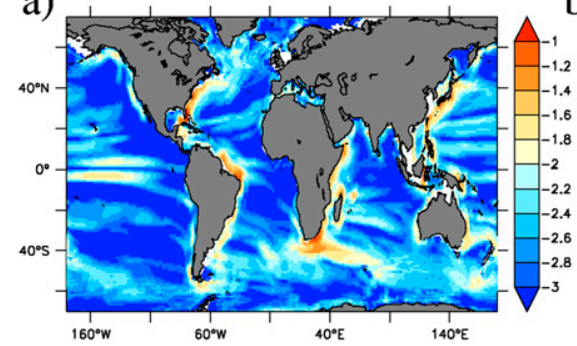

b)

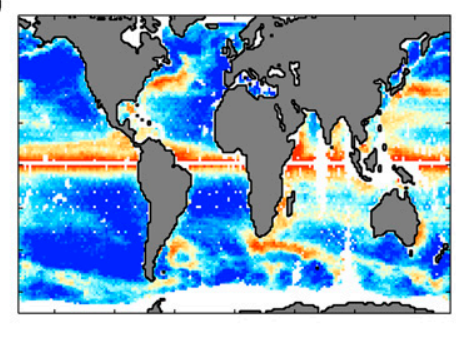

c)

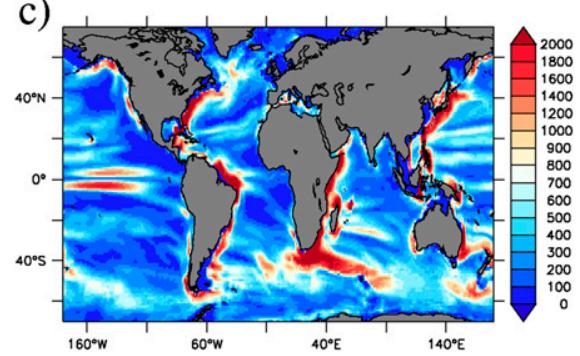

FIG. 5. $\log _{10}$-scaled $E_{\text {eddy }}\left(\mathrm{m}^{2} \mathrm{~s}^{-2}\right)$ for (a) 100-m depth in CONSIST-SURF and (b) near-surface observational estimate by Scharffenberg and Stammer (2010) on the same color scale as (a). Missing values are left white. (c) Skew diffusivity $K_{\mathrm{gm}}\left(\mathrm{m}^{2} \mathrm{~s}^{-1}\right)$ at $100-\mathrm{m}$ depth in CONSIST-SURF.

simulation. Correspondingly, von Storch et al. (2007) found a reduced power input of $1.8 \mathrm{TW}$ by fluctuating winds and flow excluding superinertial fluctuations. The difference corresponds roughly to estimates of nearinertial wind forcing by the winds at the surface (from which only about $20 \%$ will radiate into the stratified interior driving internal waves). However, both eddying models suggest a large surface energy flux of 1-2 TW into the time-varying circulation with frequencies smaller than the inertial one, which is not included in our model setup and was also not considered in observational estimates of the oceanic energy cycle (Wunsch and Ferrari 2004; Ferrari and Wunsch 2009). How much of this flux enters the interior ocean and how much is dissipated already within the surface mixed layer remains unclear. However, at least part of this additional external forcing could dissipate in the interior and would thus increase the energy input to internal waves and to small-scale turbulence and thus the energy available for mixing.

Although the wind power input to the mean circulation appears correct, we find much less mean kinetic energy compared to the eddying model, where $1.3 \times$ $10^{18} \mathrm{~J}$ are found. This difference can be related to the difference in the spatial resolution $\left(1^{\circ} \times 1^{\circ}\right.$ here compared to $0.1^{\circ} \times 0.1^{\circ}$ in the eddying model) and the increased spatial variance of the flow and to the strong viscous damping in the coarse model. Oort et al. (1989) give a rough estimate of $0.4 \times 10^{18} \mathrm{~J}$ of mean kinetic energy; more recent and precise observational estimates are not available to us.

There is also a low bias in the total eddy energy; about $10 \times 10^{18} \mathrm{~J}$ of $E_{\text {eddy }}$ is found in the eddying model, which is about an order of magnitude larger than what is suggested by our closure. The larger value is closer to the estimate of Zang and Wunsch (2001) and Wunsch and Ferrari (2004) of about $13 \times 10^{18} \mathrm{~J}$. The bulk estimate by Oort et al. (1989) of $2.5 \times 10^{18} \mathrm{~J}$ is also larger than the values in our closure. Figure 5a also suggests a low bias of the eddy energy in our closure in particular in the tropics, when compared to observational estimates of near-surface eddy energy that are shown in Fig. 5b. Part of this low bias might be explained by the missing wind forcing in our closure for mesoscale eddies. This low bias could in principle be resolved by introducing and adjusting tuning parameters in the parameterization [Eq. (6)] and by including surface forcing of mesoscale eddy energy, but we made no attempt to do so.

A rough estimate of the global internal wave energy is given by multiplying the representative mean value of $3.8 \times 10^{3} \mathrm{~J} \mathrm{~m}^{-2}$ for the canonical empirical spectrum by Munk (1981) with the surface area of the ocean, which yields about $1.4 \times 10^{18} \mathrm{~J}$ and is about 3 times larger than what is suggested by our model. Ferrari and Wunsch (2009) suggest that a fraction of $50 \%$ of the internal wave energy is related to inertial frequencies. On the other hand, von Storch et al. (2012) report an energy content of $0.4 \times 10^{18} \mathrm{~J}$ of internal waves, which are close to the inertial frequency due to the model resolution. This would suggest that the bulk value by Munk (1981) is too large. We conclude here that our simulated energy levels of total internal wave energy are not inconsistent with eddying models and observational estimates.

Observational estimates of the global production rates of eddy energy are difficult to obtain; values range from 0.3 to 1.3 TW (Ferrari and Wunsch 2009). The total conversion of $0.94 \mathrm{TW}$ of mean to eddy energy by $\delta W_{\mathrm{gm}}$ and $\bar{S}$ in the eddying model by von Storch et al. (2012) lies at the upper end of this range. Figures $6 a$ and $6 b$ show the vertically integrated transfer from mean to eddy kinetic energy and from eddy to mean kinetic energy by $\bar{S}$ in the eddying model. The total transfer is directed from the mean to eddy component with a globally integrated magnitude of $0.11 \mathrm{TW}$, which is about 4-5 times smaller than what is given by the dissipation by lateral friction in our model simulations. Locally, however, $\bar{S}$ often changes sign. Integrating only the transfer from the mean to the eddy component, that is, only the positive values of $\bar{S}$, the global value is $0.38 \mathrm{TW}$, 

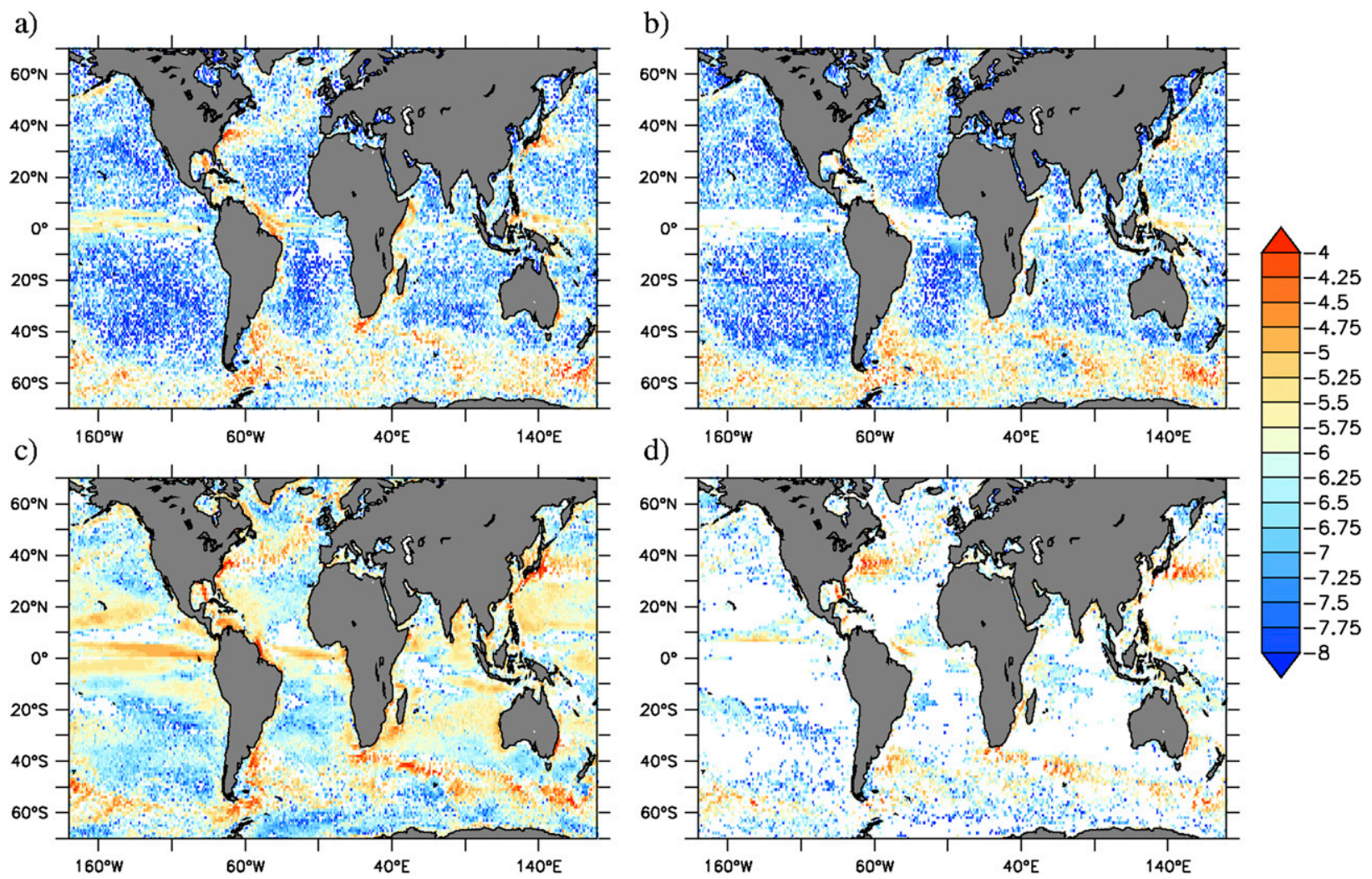

FIG. 6. (a) $\log _{10}$-scaled vertically integrated transfer of mean to eddy kinetic energy $\int_{-h}^{0} \bar{S} / \rho_{0} d z\left(\mathrm{~m}^{3} \mathrm{~s}^{-3}\right)$ in the eddying model by von Storch et al. (2012). Negative values are left white. (b) As in (a), but for negative values, that is, energy transfer from eddy to mean kinetic energy, while positive values are left white. (c) As in (a), but for eddy available potential energy $\int_{-h}^{0} \delta W_{\mathrm{gm}} / \rho_{0} d z\left(\mathrm{~m}^{3} \mathrm{~s}^{-3}\right)$. Negative values are left white. (d) As in (c), but for negative values, that is, energy transfer from eddy to mean kinetic energy.

while the backscatter from the eddy to the mean component amounts to $0.26 \mathrm{TW}$. Such a backscatter of energy is consistent with our expectation of upgradient eddy momentum fluxes and cannot be represented by harmonic friction in our model. On the other hand, the horizontal (and vertical, not shown) structure of the positive values of $\bar{S}$ correspond well with Fig. 4b; they are large near the western boundaries, along the equator and within the Southern Ocean, while low values are seen within the interior of the subtropical gyres. We thus conclude that although the unphysical viscous closure in our model yields a positive bias in the transfer to eddying motion and is not able to reproduce the backscattering, its spatial structure agrees reasonably well with the eddying model simulations.

Figures $7 \mathrm{a}$ and $7 \mathrm{~b}$ show the vertical transfer of energy by $\delta W_{\mathrm{gm}}$ from mean to eddy available potential energy in the model by von Storch et al. (2012). The globally integrated value is a transfer of $0.8 \mathrm{TW}$ from the mean to the eddy component, but locally $\delta W_{\mathrm{gm}}$ also can become negative; the global transfer separates into $1.1 \mathrm{TW}$ by positive values and 0.3 TW by negative values. However, regions with backscattering from the eddy to the mean component are much smaller than for $\bar{S}$ consistent with our expectation of eddy production by baroclinic instability. The total value of $\delta W_{\mathrm{gm}}$ is significantly larger in the eddying model than implied by our model, ${ }^{6}$ but almost compensated by the positive bias in $\bar{S}$. Except for the regions with negative $\delta W_{\mathrm{gm}}$, the horizontal (and vertical, not shown) structure of $\delta W_{\mathrm{gm}}$ corresponds again well with Fig. $4 \mathrm{a}$.

\section{f. Internal wave dissipation and vertical diffusivities}

Figures $7 \mathrm{a}$ and $7 \mathrm{~b}$ show the vertical diffusivity $K$ along $30^{\circ} \mathrm{W}$ in STANDARD and in WAVE, respectively. In STANDARD, the interior diffusivity is given by the minimal threshold for $E_{\mathrm{tke}}$ by $K=\sqrt{E_{\min }} L$. Since $L$ also depends only on $N$ and $E_{\min }$, the vertical structure in $K$ seen in Fig. 7 is therefore only due the vertical dependency of the stratification $N$. As discussed in Olbers

\footnotetext{
${ }^{6} \mathrm{We}$ could reduce $\delta W_{\mathrm{gm}}$ in our model by adjusting the eddy closure and thus $K_{\mathrm{gm}}$ that would yield a way to validate the eddy closure, but we made no attempt to do so.
} 
a)

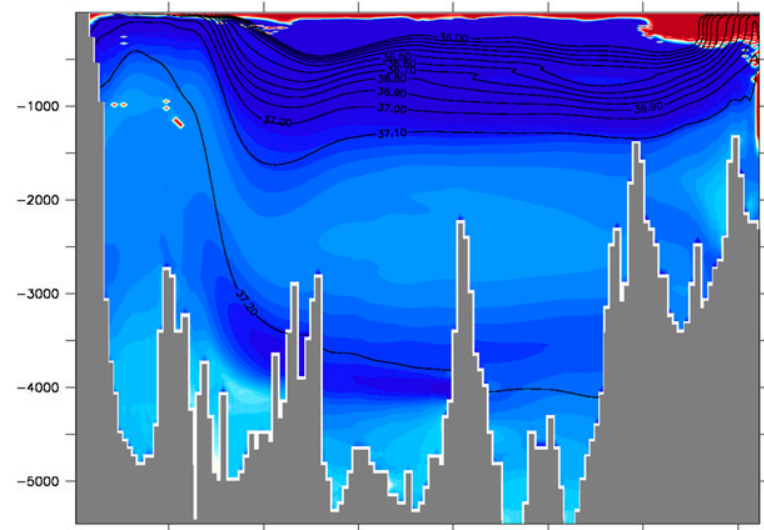

c)

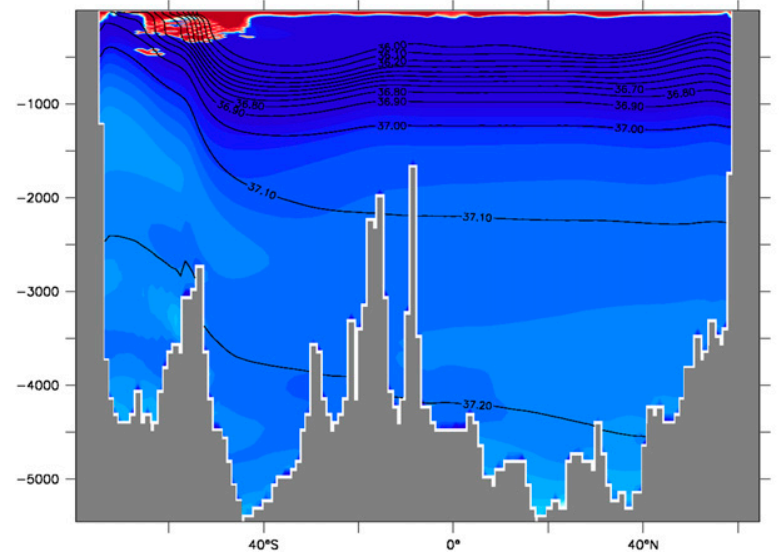

b)

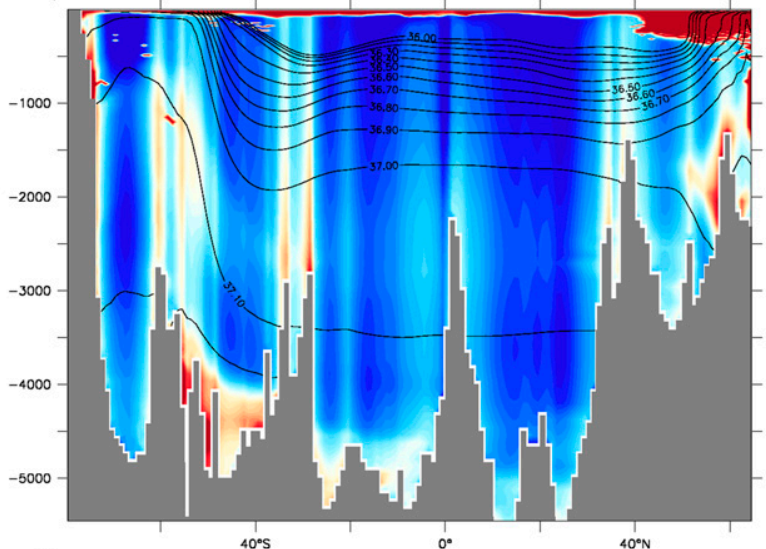

d)

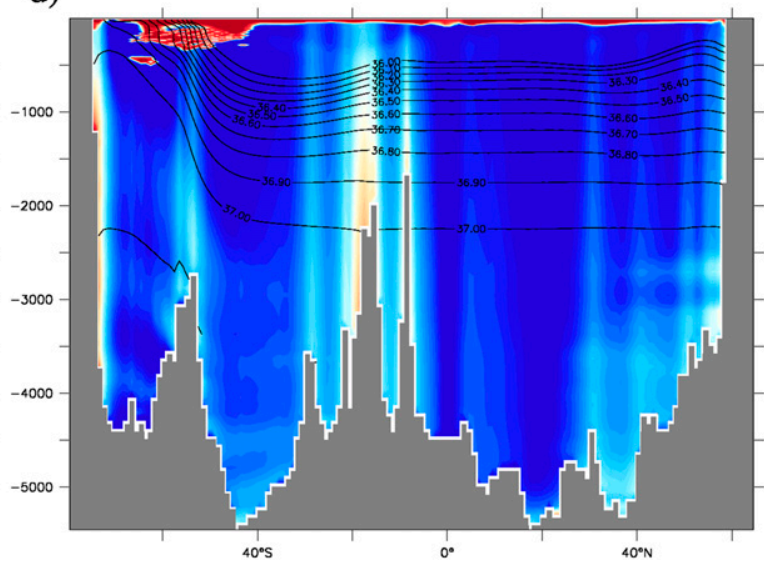

FIG. 7. (a) $\log _{10}$-scaled vertical diffusivity $K\left(\mathrm{~m}^{2} \mathrm{~s}^{-1}\right)$ along (a),(b) $30^{\circ}$ and (c),(d) $140^{\circ} \mathrm{W}$ in (left) STANDARD and (right) WAVE. Also shown are isopycnals ( $\sigma_{2}$, solid lines).

and Eden (2013), the internal wave parameterization gives more realistic diffusivities that are enhanced near the bottom due to the tidal forcing and very small within the thermocline. Figure $7 \mathrm{~b}$ is in fact very similar to Fig. 5c in Olbers and Eden (2013), where $K$ was diagnosed using the internal wave parameterization with identical tidal forcing, similar to near-inertial wave forcing and the observed stratification. In the subtropical thermocline, $K$ stays below $10^{-5} \mathrm{~m}^{2} \mathrm{~s}^{-1}$, while poleward of about $30^{\circ}$ the diffusivity increases. In particular in the Southern Ocean, $K$ is enhanced to values of about $10^{-4} \mathrm{~m}^{2} \mathrm{~s}^{-1}$. The same can be seen at other longitudes. Figures $7 \mathrm{c}$ and $7 \mathrm{~d}$ show the vertical diffusivity $K$ along $140^{\circ} \mathrm{W}$ in STANDARD and in WAVE. The deep values of $K$ in WAVE are again enhanced over locations with enhanced tidal forcing, as for instance at $20^{\circ}-10^{\circ} \mathrm{S}$ along the section, while $K$ stays below $10^{-5} \mathrm{~m}^{2} \mathrm{~s}^{-1}$ elsewhere. In the Southern Ocean, $K$ stays smaller than at $30^{\circ} \mathrm{W}$ since tidal forcing is also smaller here.

Adding also the mesoscale eddy dissipation as the forcing of the internal wave field, $K$ increases again, as seen in Fig. 8, showing $K$ along $30^{\circ} \mathrm{W}$ for CONSIST-BOT, CONSIST-INT, and CONSISTSURF. While $K$ within the thermocline of the subtropical ocean stays small, it is now enhanced at depth and in particular in the Southern Ocean where values up to $10^{-3} \mathrm{~m}^{2} \mathrm{~s}^{-1}$ below $1000-\mathrm{m}$ depth are met in CONSIST-BOT and CONSIST-INT. In CONSISTSURF, $K$ is much smaller in the Southern Ocean, but still enhanced compared to WAVE. The reason for the large values of $K$ in the Southern Ocean is the large dissipation of mesoscale eddy energy, which can also be seen in Fig. 4c. At $30^{\circ} \mathrm{W}, K$ in CONSIST-BOT and CONSIST-INT are also similar to GM-INT (not shown) such that the exact value of the skewness diffusivity $K_{\mathrm{gm}}$ appears to play a minor role for the dissipation and resulting vertical diffusivity $K$. At $140^{\circ} \mathrm{W}$, GM-INT show very large diffusivities near the bottom in the Southern Ocean, related to almost vanishing stratification, an artifact apparently related to the fixed skew (GM) diffusivity $K_{\mathrm{gm}}$ and excessive available potential energy release. 
a)

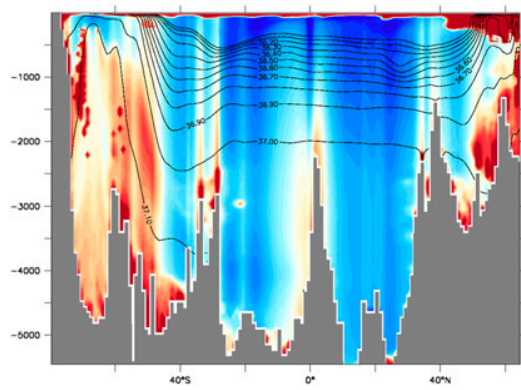

b)

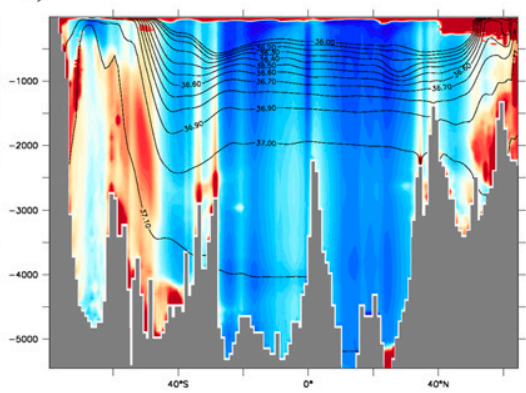

c)

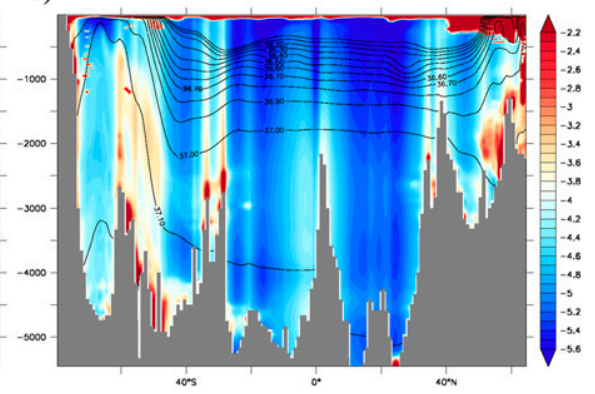

FIG. 8. (a) $\log _{10}$-scaled vertical diffusivity $K\left(\mathrm{~m}^{2} \mathrm{~s}^{-1}\right)$ along $30^{\circ} \mathrm{W}$ in (a) CONSIST-BOT, (b) CONSIST-INT, and (c) CONSIST-SURF. Also shown are isopycnals $\left(\sigma_{2}\right.$, solid lines).

Although a detailed comparison with observational estimates of $K$ was not made in this study, the diffusivities in CONSIST-BOT and CONSIST-INT appear to be larger in the Southern Ocean than observations suggest (e.g., Wu et al. 2011; Sheen et al. 2013). Largest values of $K$ can be found at $30^{\circ} \mathrm{W}$, but they are also elsewhere enhanced in the Southern Ocean compared to the rest of the ocean, which can also be seen in Fig. 9, showing $K$ at 2000-m depth. Since the global production rates of mesoscale eddies appear reasonable compared to eddying model simulations, which also hold for the Southern Ocean and the spatial structure shown in Fig. 4c, and since a significant transfer of eddy energy out of the Southern Ocean is not likely, it follows that the transfer of the mesoscale energy to internal waves by interior loss of balance and lee-wave generation is too large in CONSIST-BOT and CONSIST-INT. Dissipating the larger part of the eddy energy in the mixed layer in CONSIST-SURF, where it is not available for interior mixing, appears to be in better agreement with observational estimates.

\section{g. Meridional transports}

The different amounts of energy available for interior mixing and the resulting different vertical diffusivities lead in turn to differences in the meridional overturning circulation. Figure 10 shows the isopycnal streamfunction $\Psi$ for the global meridional overturning circulation (MOC) in STANDARD and WAVE. To calculate $\Psi$, we have averaged the depth and meridional transport in 160 isopycnal layers for the last $10 \mathrm{yr}$ of the simulation, using the potential density referenced to 2000 dbar. The streamfunction $\Psi$ is defined as the zonally integrated meridional transport in each layer and is shown in Fig. 10 as a function of the zonally averaged isopycnal layer depth, which is then identical to the quasi-Stokes streamfunction defined by McDougall and McIntosh (2001) [cf. also the discussion in Olbers et al. (2012) and Viebahn and Eden (2012)]. The meridional layer transport includes both the mean and eddy-driven advection velocities. Also shown in Fig. 10 are three selected zonally averaged layer depths that are identical to isolines of the modified (potential) density by McDougall and McIntosh (2001). We use here a reference level of $2000 \mathrm{dbar}$ for the potential density, but tests with 3000 and 4000 dbar show virtually no difference. On the other hand, the deep flow field becomes much different when we use the surface as reference, while this is not the case for the surface circulation (not shown). We use the isopycnal streamfunction $\Psi$ instead of the conventional streamfunction using the zonally averaged Eulerian mean velocity, since the former more a)

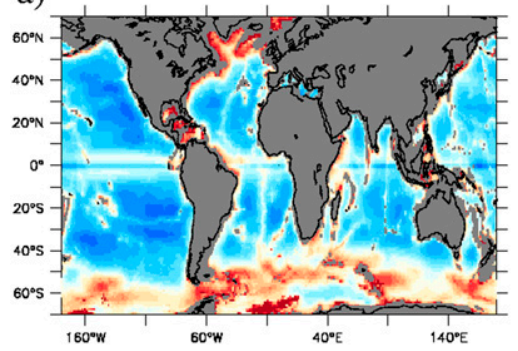

b)

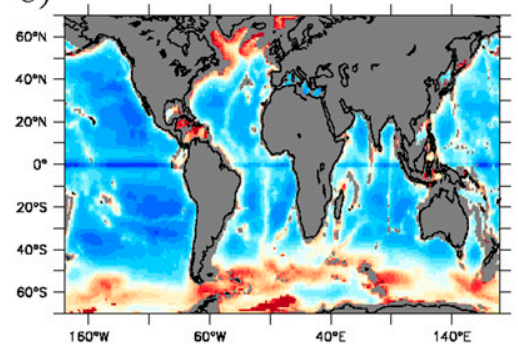

c)

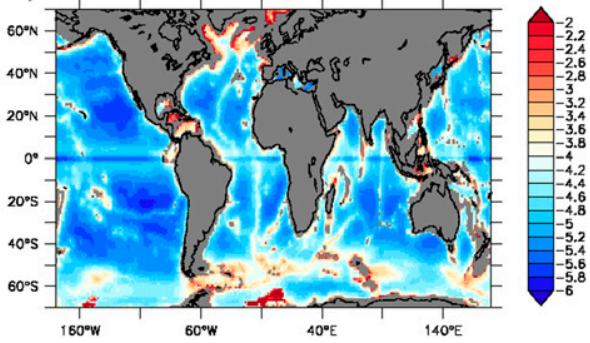

FIG. 9. (a) $\log _{10}$-scaled vertical diffusivity $K\left(\mathrm{~m}^{2} \mathrm{~s}^{-1}\right)$ at 2000-m depth in (a) CONSIST-BOT, (b) CONSIST-INT, and (c) CONSIST-SURF. 
a)

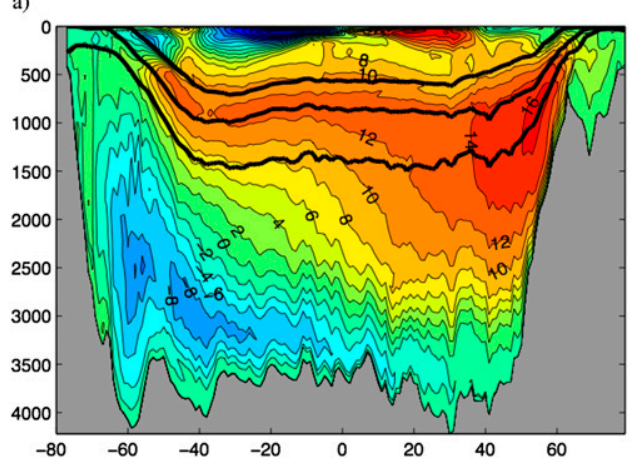

b)

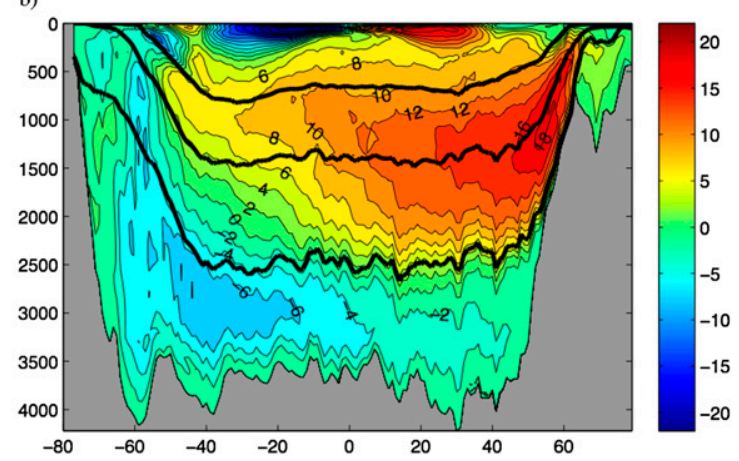

FIG. 10. (a) Isopycnal global MOC streamfunction $\Psi(\mathrm{Sv})$ as a function of the mean isopycnal layer depth for STANDARD. See text for definition of this streamfunction. (b) As in (a), but for WAVE. Thick solid lines denote isolines $36,36.5$, and $36.65 \mathrm{~kg} \mathrm{~m}^{-3}$ of the modified potential density referenced to $2000 \mathrm{dbar}$.

consistently describes the flow field acting on mean tracers and density as discussed in, for example, McDougall and McIntosh (2001), Olbers et al. (2012), and Viebahn and Eden (2012). Spurious cells like the so-called Deacon cell in the Southern Ocean complicate the interpretation of the conventional Eulerian mean streamfunction, but do not show up in $\Psi$. Since the integration is taken along latitudinal circles, $\Psi$ cannot, however, account for standing zonal eddy circulation, as discussed by Viebahn and Eden (2012), but we regard this artifact as less essential for the present discussion.

As prominent features, $\Psi$ shows a clockwise cell between about 500- and 2000-m depth in all model simulations and an anticlockwise bottom cell below. The deep cell is related to the circulation of deep and intermediate water masses. The deep water masses are formed in the subpolar North Atlantic flowing at depth southward, reaching the surface again in the Southern Ocean, where equatorward Ekman flow leads to subduction of intermediate water masses, establishing the northward return flow of the deep cell within and below the thermocline. The bottom cell is related to the circulation of bottom water masses that are formed in the Southern Ocean and flow equatorward at the bottom, where they upwell into the deep water masses by diapycnal mixing. In addition to the deep and bottom cells, wind-driven, near-surface cells symmetric to the equator can also be seen in $\Psi$ in all simulations.

Since mixing is supposed to be important for the transports in the deep ocean, we focus here on the deep and bottom cell. In STANDARD, the deep cell has a maximal amplitude of 15.5 Sverdrups $(\mathrm{Sv} ; 1 \mathrm{~Sv} \equiv$ $10^{6} \mathrm{~m}^{3} \mathrm{~s}^{-1}$ ) at $48^{\circ} \mathrm{N}$, and the bottom cell a maximal amplitude of $8.2 \mathrm{~Sv}$ at $32^{\circ} \mathrm{S}$. This is, in particular for the bottom cell, much weaker than what observational estimates suggest. Lumpkin and Speer (2007) report from inversions of hydrographic sections $17.2 \pm 3.3 \mathrm{~Sv}$ at $48^{\circ} \mathrm{N}$ for the deep cell and $20.9 \pm 6.7 \mathrm{~Sv}$ at $32^{\circ} \mathrm{S}$ for the bottom cell in the global overturning. These numbers are given for the Eulerian mean velocity, while in our model we (correctly) use the transports by the sum of Eulerian mean and eddy-driven advection velocity. We note that in particular in the Southern Ocean, the eddy-driven advection velocity can become as large as the Eulerian mean velocity and thus is a large component of the total transport. The comparison with the observational estimates is therefore difficult in particular in the Southern Ocean, but it nevertheless appears that STANDARD has a too weak bottom cell.

In WAVE, the deep cell increases in the north to an amplitude of $17.0 \mathrm{~Sv}$ at $48^{\circ} \mathrm{N}$, while the bottom slightly weakens to $6.6 \mathrm{~Sv}$ at $32^{\circ} \mathrm{S}$. The main difference between STANDARD and WAVE in the deep cell are much larger upward or diapycnal transports north of $30^{\circ} \mathrm{S}$ in WAVE, while in STANDARD the flow is more directed along isopycnals with weaker diapycnal transports. Since the simulations are in steady state, the diapycnal transports are locally generated by diapycnal mixing. The upwelling in WAVE of about $8 \mathrm{~Sv}$ takes place almost exclusively in the Pacific and Indian Oceans. This can be seen in Fig. 11a, showing the streamfunction $\Psi$ in WAVE calculated for the Atlantic Ocean only, and in Fig. 11b, showing $\Psi$ calculated for the Pacific and Indian Oceans only. In the Atlantic Ocean, the deep cell shows very small upwelling. The amplitude of the deep cell in the Atlantic Ocean is in WAVE at $17.8 \mathrm{~Sv}$ at $48^{\circ} \mathrm{N}$, which is about $2 \mathrm{~Sv}$ larger than in STANDARD (see Table 3). The value in WAVE is comparable to the observational estimate by Lumpkin and Speer (2007) of $16.3 \pm 2.7 \mathrm{~Sv}$ at $48^{\circ} \mathrm{N}$, but still smaller at $24^{\circ} \mathrm{N}$ where the observational estimate is $18.0 \pm 2.5 \mathrm{~Sv}$. The observational estimate of the mean strength of the deep Atlantic cell of $18.6 \mathrm{~Sv}$ at $26.5^{\circ} \mathrm{N}$ from the Rapid Climate Change (RAPID) array 
a)

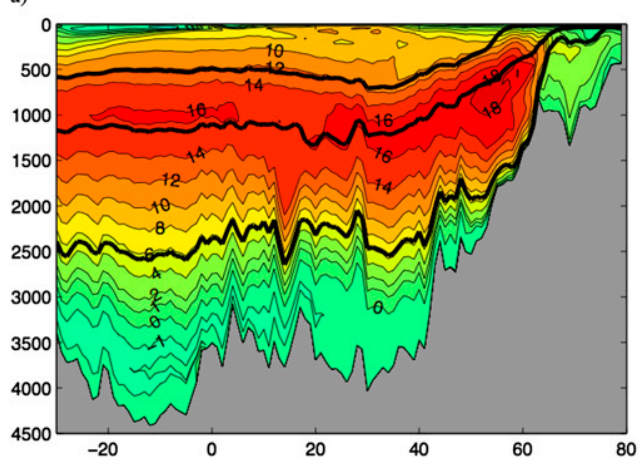

b)

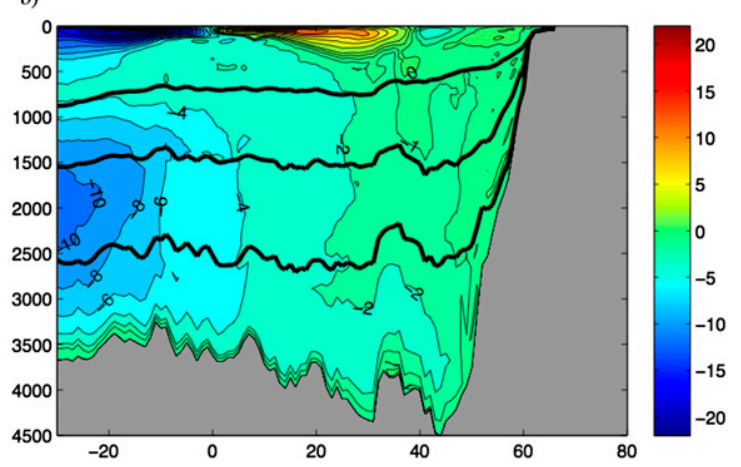

FIG. 11. As in Fig. 10, but for $\Psi$ in WAVE for the (a) Atlantic Ocean and (b) Pacific and Indian Oceans only.

(Cunningham et al. 2007) agrees well with the estimate of Lumpkin and Speer (2007).

The bottom cell in the Pacific and Indian Oceans, on the other hand, shows a mixing-driven upwelling of $11.7 \mathrm{~Sv}$ at $32^{\circ} \mathrm{S}$ in WAVE, while there is $9.9 \mathrm{~Sv}$ at $32^{\circ} \mathrm{S}$ in STANDARD (not shown). This is both much smaller than the observational estimates by Lumpkin and Speer (2007), who report $12.4 \pm 2.6 \mathrm{~Sv}$ and $10.3 \pm 5.1 \mathrm{~Sv}$ of bottom water entering the Indian and Pacific Oceans, respectively (we give the sum of both in Table 3). Using only the Eulerian mean velocity instead of including also the eddy-driven velocity does not yield any difference in the low bias in the model transport. The streamfunction averaged at constant geopotentials also shows a low bias in the Pacific-Indian bottom cell. On the other hand, the bottom cell quickly ceases going northward in Fig. 11b, which is also seen in the observations; at $24^{\circ} \mathrm{N}$, it decreases to $5.1 \pm 3.1 \mathrm{~Sv}$, and at $48^{\circ} \mathrm{N}$, it decreases to $2.2 \pm$ 1.5 Sv (Lumpkin and Speer 2007). In summary, we see that the increased energy available for mixing and the larger diffusivities in WAVE generates increased upwelling of bottom water in the Pacific and Indian Oceans, while in the Atlantic Ocean an increased strength of the deep cell can be seen, but no increased upwelling there. The bottom cells in the Pacific/Indian Oceans appear to be weak.
Figure 12 shows $\Psi$ for the Atlantic and the Pacific/ Indian Oceans in the more energetic experiment CONSIST-SURF. Because of the increased diapycnal mixing, the deep cell in the Atlantic Ocean is further increased to $19.4 \mathrm{~Sv}$ at $48^{\circ} \mathrm{N}$, while the bottom cell in the Pacific/Indian Oceans is also increased, but still shows a low bias compared to the observations. The differences to CONSIST-INT and CONSIST-BOT are relatively small; the deep cell in the Atlantic Ocean is slightly stronger in CONSIST-INT and CONSIST-BOT, while the bottom cell in the Pacific-Indian Ocean is slightly stronger in CONSIST-BOT and similar in CONSIST-INT (see Table 3). The differences are small since the vertical diffusivities in both experiments are also similar. This is because the internal wave closure is rather efficient in propagating the injected energy in the vertical, such that the dependency on the localization of the energy input in the vertical-at the bottom or in the interior-appears to be weak as long as the magnitude is identical.

Of direct importance in a climate model is the heat transport of the ocean component, which we thus show in Fig. 13. The maximum of the global northward heat transport at about $20^{\circ} \mathrm{N}$ is as expected, increasing with increasing deep overturning cells in the Atlantic Ocean, from $1.30 \mathrm{PW}$ in STANDARD to $1.43 \mathrm{PW}$ in CONSIST-INT.

TABLE 3. Transport from observations and experiments (Sv). Observations are from Lumpkin and Speer (2007). The first four columns $48^{\circ} \mathrm{N}$ to $32^{\circ} \mathrm{S}$ refer to the deep cell in the Atlantic Ocean, the following columns $32^{\circ} \mathrm{S}$ to $48^{\circ} \mathrm{N}$ refer to the magnitude of the bottom cell in the Pacific Ocean.

\begin{tabular}{|c|c|c|c|c|c|c|c|}
\hline & $48^{\circ} \mathrm{N}$ & $24^{\circ} \mathrm{N}$ & $11^{\circ} \mathrm{S}$ & $32^{\circ} \mathrm{S}$ & $32^{\circ} \mathrm{S}$ & $24^{\circ} \mathrm{N}$ & $48^{\circ} \mathrm{N}$ \\
\hline Observations & $16.3 \pm 2.7$ & $18.0 \pm 2.5$ & $16.2 \pm 3.0$ & $12.4 \pm 2.6$ & $22.7 \pm 7.7$ & $5.1 \pm 3.1$ & $2.2 \pm 1.5$ \\
\hline STANDARD & 15.6 & 14.0 & 14.1 & 14.2 & 9.9 & 2.5 & 0.3 \\
\hline WAVE & 17.8 & 16.2 & 16.1 & 15.7 & 11.7 & 3.0 & 1.7 \\
\hline GM-INT & 19.0 & 17.0 & 16.9 & 16.5 & 12.3 & 2.7 & 1.7 \\
\hline CONSIST-INT & 20.6 & 18.9 & 18.3 & 17.7 & 12.1 & 2.6 & 1.5 \\
\hline CONSIST-BOT & 20.9 & 18.6 & 18.0 & 17.4 & 13.5 & 2.7 & 1.5 \\
\hline CONSIST-SURF & 19.4 & 18.0 & 17.8 & 17.3 & 12.1 & 2.5 & 1.5 \\
\hline
\end{tabular}



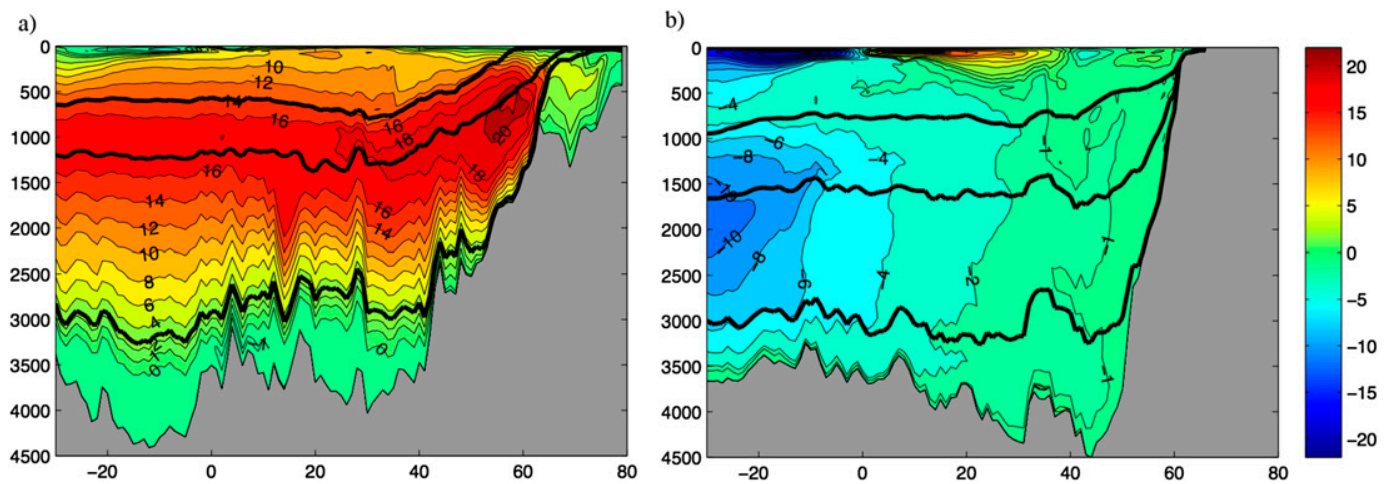

FIG. 12. As in Fig. 11, but for CONSIST-SURF.

The latter is still lower than what observational estimates suggest but similar to comparable ocean models (Griffies et al. 2009).

\section{h. Water mass age}

It is clear that the differences in the MOC lead to different ventilation rates of the deep ocean in the model simulations. Figure 14a shows an observational estimate of water mass age at 3000-m depth using the method of Matsumoto (2007). We use a gridded climatology of natural $\delta^{14} \mathrm{C}$ by Key et al. (2004) (this dataset can be downloaded at http://cdiac.esd.ornl.gov). Following Matsumoto (2007), the different preformed surface values of $\delta^{14} \mathrm{C}$ are corrected by using $\mathrm{PO}_{4}^{*}$ to identify the fraction of deep and bottom water masses of the water parcel. The term $\mathrm{PO}_{4}^{*}$ is the observed phosphate concentration $\mathrm{PO}_{4}$ corrected by the apparent oxygen utilization due to remineralization of organic matter. The respective fractional surface values of $\delta^{14} \mathrm{C}$ values of deep and bottom water masses are subtracted from the $\delta^{14} \mathrm{C}$ value of the water parcel. Finally, the $\delta^{14} \mathrm{C}$ values are converted to water mass age using $t=$ $8033 \ln \left(1+\delta^{14} \mathrm{C} / 1000\right)(\mathrm{yr})$.

We compare this observational estimate of water mass age with an idealized age tracer, which was integrated in the model simulations for $1000 \mathrm{yr}$ using the identical advection scheme and diffusivities as the other tracers. Figure 14b shows the age tracer in STANDARD at 3000-m depth. In the Southern Ocean, the water mass age is much higher in STANDARD compared to the observational estimates indicating weak ventilation. Figures $14 \mathrm{c}$ and $14 \mathrm{~d}$ show the age at $3000-\mathrm{m}$ depth in WAVE and CONSIST-SURF with larger mixing rates and stronger bottom cell in the Pacific/Indian Oceans. In fact, these experiments are in better agreement with the observational estimates in the Southern Ocean, although the observational estimates still suggest more ventilation and smaller water mass age.

\section{Remaining energy imbalances}

A conceptual problem constructing energetically consistent ocean models is that of energy sources and sinks due to the nonlinear equation of state. Based on numerical model simulations, Urakawa and Hasumi (2010) and Urakawa et al. (2013) show that those sources and sinks can reach $O(10) \%$ of the leading-order term $g \bar{\rho} \bar{w}$ in the potential energy equation, although they also interpreted effects due to compressibility as irreversible exchange with the parameterized forms of energy. Effects due to compressibility do not show up, considering mean dynamic enthalpy instead of mean gravitational potential energy. Based on the consistent definition of total energy in a Boussinesq ocean model given by Eden (2014, manuscript submitted to J. Phys. Oceanogr.), we will show in a later study how these terms can be included to obtain an exactly closed energy budget of the model.

Since the terms are related to the eddy fluxes of $\theta$ and $S$, we might tend to direct them to the forms of

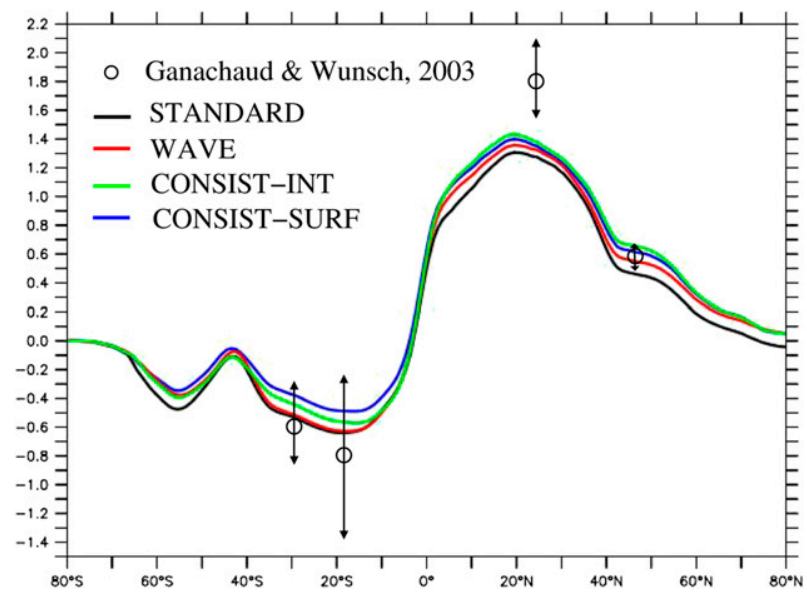

FIG. 13. Northward heat transport (PW) in four different experiments. Also shown are observational estimates from Ganachaud and Wunsch (2003). 
a)

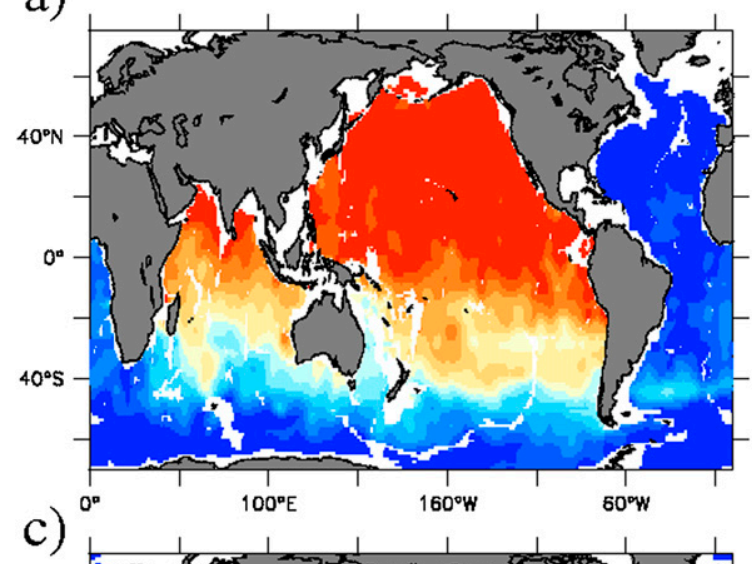

c)

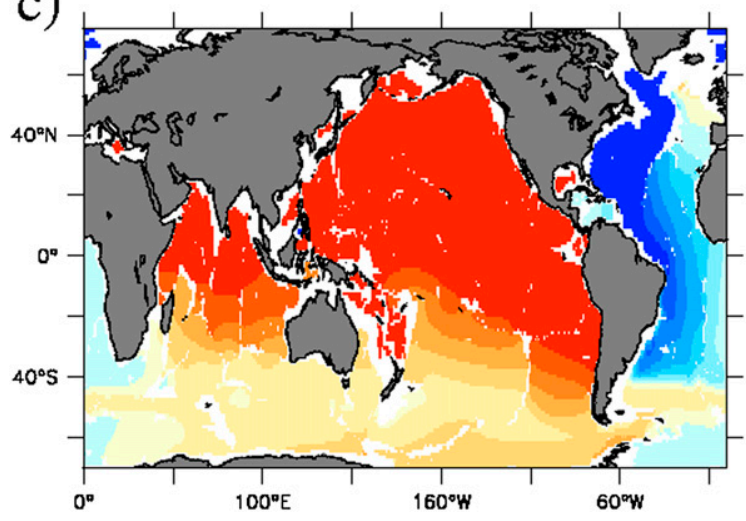

b)

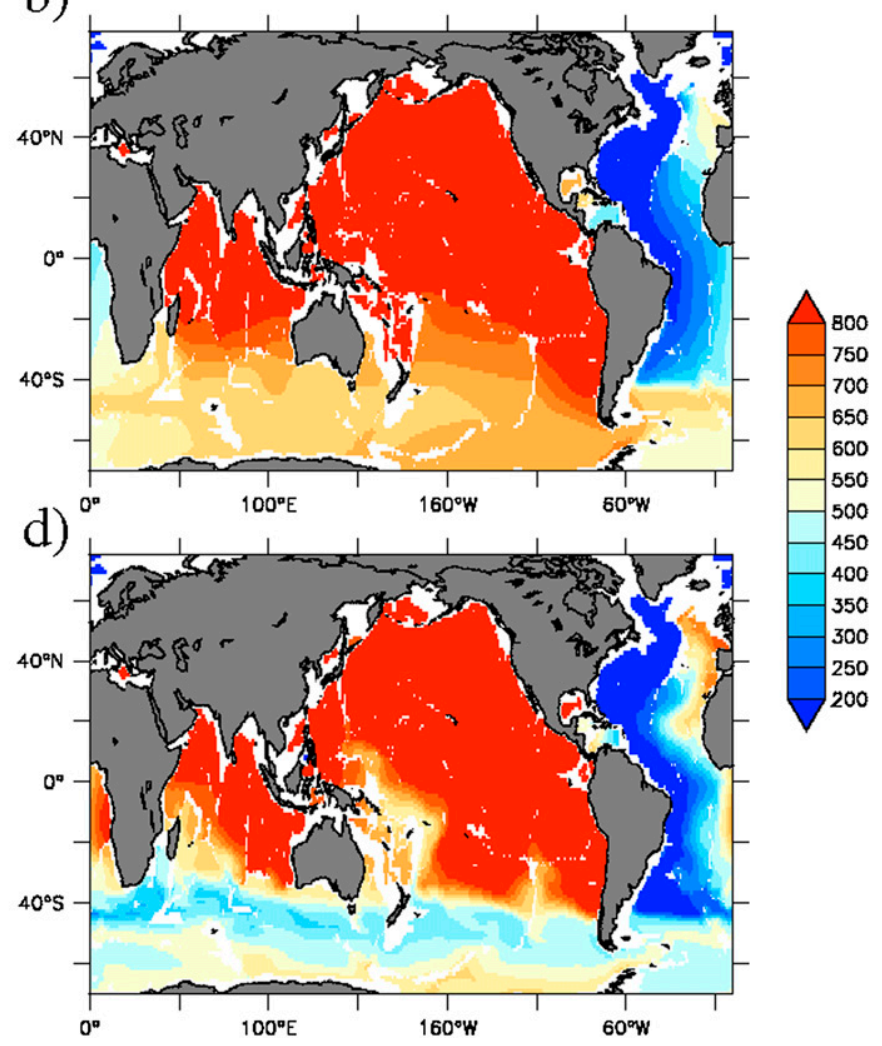

FIG. 14. (a) Estimate of water mass age at 3000-m depth in years. See text for more details. (b) Mean age tracer at 3000-m depth in STANDARD. (c) As in (a), but for WAVE. (d) As in (a), but for CONSIST-SURF.

parameterized energies, as anticipated by Urakawa and Hasumi (2010) and Urakawa et al. (2013). On the other hand, we might find no reason for those exchange terms to show up in equations of the parameterized energies. This is, for instance, the case for the turbulent small-scale kinetic energy, where exactly $g \overline{\rho^{\prime} w^{\prime}}$ shows up and no additional effect due to the nonlinear equation of state. The same holds for internal wave or mesoscale eddy energy. Furthermore, it appears inconsistent to turn reversible energy exchanges due to the compressibility into irreversible exchanges with the parameterized forms of energy as anticipated by Urakawa and Hasumi (2010) and Urakawa et al. (2013). A pragmatic solution of this conceptual problem is to calculate the (irreversible) sources and sinks due to the nonlinear equation of state during the model integration and to simply add them to the energy equations of the parameterized forms of energy. In the present study, however, we simply ignore these effects and discuss the results of an approximately consistent ocean model and leave the discussion of an exactly consistent model to a later study.

Another problem for energetically consistent ocean models is spurious imbalances due to numerical artifacts. Consistent numerical equivalents of the forcing terms in the individual energy equations are necessary to eliminate the spurious numerical imbalances. For instance, the dissipation of mean kinetic energy by vertical friction that enters the TKE equation has to exactly match the amount of mean kinetic energy that is dissipated by the numerical discretization in the mean momentum equation. It was shown by Burchard (2002) that even in a one-dimensional example both the spatial and the temporal discretization of friction and dissipation needs care to obtain an energetically consistent numerical scheme. The schemes that we use here in the closures might not be energetically consistent on the numerical level. Furthermore, we have not checked to which extent other discretizations in the model for, for example, the Coriolis term and the momentum advection conserve mean kinetic and potential energy. To resolve the numerical biases, a substantial change to the numerical code appears necessary, which we leave for a later study.

\section{Summary and discussion}

We have explored in this study the possibility of constructing an energetically consistent, realistic ocean 
model. In such a consistent model, a full account of the energy cycle of the ocean in principle becomes possible. In particular, the amount of energy for mixing in the interior ocean is controlled and depends only on the external forcing. We found in our approximately consistent model simulations that from the total energy input of about $4 \mathrm{TW}$ into the ocean, only $0.3-0.4 \mathrm{TW}$ are used to drive the large-scale mean circulation by interior mixing of density due to breaking internal waves. This is much smaller than the direct wind forcing of the surface flow of about 1.8 TW. A fraction of the flux into the internal wave field comes from the dissipation of mesoscale eddies, while the dominant part comes from tidal forcing (plus a minor part from near-inertial waves). This fraction $(10 \%-40 \%)$ depends on the mechanism and thus vertical localization of eddy dissipation in our sensitivity experiments.

Central to the effort to build an energetically consistent ocean model is the recently developed energetically based closure for internal wave breaking by Olbers and Eden (2013) and Eden and Olbers (2014). It links the sources of energies for internal waves, propagates the energy laterally and vertically, and supplies it to smallscale turbulence. Important sources are near-inertial wave forcing in the surface mixed layer and the tidal forcing at the bottom, but there might be other sources of internal waves, such as the direct coupling between surface waves and internal waves that has not received the necessary attention so far. Furthermore, the lateral propagation of low vertical modes differs from the rest of the internal wave spectrum, which is not accounted for in the closure by Olbers and Eden (2013), such that an internal wave model with more compartments as suggested by Eden and Olbers (2014) might provide a more realistic energy and mixing rate distribution.

The dissipation of balanced flow is a potentially major source for internal wave energy, which we have implemented only in a premature way since there is not much known about this process. Several mechanisms have been proposed so far, that is, lee-wave generation by flow over topography (Bell 1975; Nikurashin and Ferrari 2011), unbalanced instabilities at finite Rossby numbers (Molemaker et al. 2005), and Lighthill radiation (Ford et al. 2000) or simply a direct kinetic energy cascade to smaller scales, which appears to be favored at large Rossby numbers (Capet et al. 2008b; Molemaker et al. 2010). The role of a turbulent bottom boundary layer and the implied dissipation by bottom friction on the geostrophic flow and direct energy transfer to small-scale turbulence was excluded in our model setup, but might also be important. A detailed assessment and quantification of the relative importance of each mechanism and ways for parameterization are currently missing, but are mandatory for a consistent description of the energetics and mixing in the ocean. The same applies to the treatment of lateral dissipation, that is, harmonic or biharmonic friction. We have tested three different scenarios for the dissipation of balanced flow. Assuming that the mesoscale energy dissipates entirely by the generation of lee waves at the bottom (CONSIST-BOT) leads to very large diffusivities in the Southern Ocean that appear to contradict observational estimates of mixing rates. The same holds for the scenario of interior loss of balance (CONSIST-INT), while dissipating a large part of the mesoscale energy in the mixed layer and the rest at the bottom (CONSIST-SURF) yields diffusivities that appear in better agreement with observational estimates. On the other hand, a detailed comparison with available observations was not made in this study.

The analysis of the energy reservoirs revealed a low bias in the mesoscale eddy energy and in the mean kinetic energy. The latter is related to too strong viscous damping, while the former bias could in principle be removed by introducing and adjusting additional tuning parameters in the closure for mesoscale eddy energy without much consequences on the energy transfers and thus on vertical diffusivities. However, we have not accounted for the wind forcing of mesoscale eddies. This additional forcing might also be partly available for interior mixing (depending on the dissipation scenario). Although we found low biases in mean and eddy kinetic energy, we are confident that we have reliable estimates of external energy forcing and internal transfers. We thus have a reliable estimate of the energy supplied to the large-scale circulation by density mixing.

The meridional transports in the model are getting stronger in the more energetic consistent model versions because of increased mixing both in the deep cell in the Atlantic and bottom cell in the Pacific/Indian Oceans. The energy input by tides to the internal wave field is mostly responsible for this increased interior mixing. In the Pacific Ocean, however, a low bias in the magnitude compared to observational estimates is still present. This means that additional energy supply is needed in the Pacific/Indian Oceans to drive a stronger bottom cell. A possible energy source that we have excluded in our model configuration is geothermal heating, which is known to be strong in the Pacific Ocean and to generate a significant circulation (Stommel 1982; Adcroft et al. 2001; Emile-Geay and Madec 2009) and which might explain the low bias in the bottom cell of the Indian/ Pacific Oceans in the model.

Acknowledgments. The authors thank two anonymous reviewers and Steve Griffies for helpful comments 
that improved this manuscript. We also thank J.-S. von Storch for providing the data of the eddying model.

\section{REFERENCES}

Adcroft, A., J. R. Scott, and J. Marotzke, 2001: Impact of geothermal heating on the global ocean circulation. Geophys. Res. Lett., 28, 1735-1738, doi:10.1029/2000GL012182.

Barnier, B., L. Siefridt, and P. Marchesiello, 1995: Thermal forcing for a global ocean circulation model using a three year climatology of ECMWF analysis. J. Mar. Syst., 6, 363-380, doi:10.1016/0924-7963(94)00034-9.

Batchelor, G. K., 1982: The Theory of Homogeneous Turbulence. Cambridge University Press, $197 \mathrm{pp}$.

Bell, T. H., 1975: Topographically generated internal waves in the open ocean. J. Geophys. Res., 80, 320-327, doi:10.1029/ JC080i003p00320.

Blanke, B., and P. Delecluse, 1993: Variability of the tropical Atlantic Ocean simulated by a general circulation model with two different mixed-layer physics. J. Phys. Oceanogr., 23, 1363-1388, doi:10.1175/1520-0485(1993)023<1363:VOTTAO>2.0.CO;2.

Bretherton, F., 1966: Critical layer instability in baroclinic flows. Quart. J. Roy. Meteor. Soc., 92, 325-334, doi:10.1002/ qj.49709239302.

Burchard, H., 2002: Energy-conserving discretisation of turbulent shear and buoyancy production. Ocean Modell., 4, 347-361, doi:10.1016/S1463-5003(02)00009-4.

Capet, X., J. McWilliams, M. Molemaker, and A. Shchepetkin, 2008a: Mesoscale to submesoscale transition in the California Current System. Part I: Flow structure, eddy flux, and observational tests. J. Phys. Oceanogr., 38, 29-43, doi:10.1175/ 2007JPO3671.1.

,,--- , and $\longrightarrow, 2008 \mathrm{~b}$ : Mesoscale to submesoscale transition in the California Current System. Part III: Energy balance and flux. J. Phys. Oceanogr., 38, 2256-2269, doi:10.1175/2008JPO3810.1.

Cummins, P. F., G. Holloway, and A. E. Gargett, 1990: Sensitivity of the GFDL ocean general circulation model to a parameterization of vertical diffusion. J. Phys. Oceanogr., 20, 817-830, doi:10.1175/1520-0485(1990)020<0817:SOTGOG > 2.0.CO;2.

Cunningham, S. A., and Coauthors, 2007: Temporal variability of the Atlantic meridional overturning circulation at $26.5^{\circ} \mathrm{N}$. Science, 317, 935-938, doi:10.1126/science.1141304.

Eden, C., 2010a: Anisotropic rotational and isotropic residual isopycnal mesoscale eddy fluxes. J. Phys. Oceanogr., 40, 25112524, doi:10.1175/2010JPO4397.1.

, 2010b: Parameterising meso-scale eddy momentum fluxes based on potential vorticity mixing and a gauge term. Ocean Modell., 32, 58-71, doi:10.1016/j.ocemod.2009.10.008.

—_, and R. J. Greatbatch, 2008a: Diapycnal mixing by mesoscale eddies. Ocean Modell., 23, 113-120, doi:10.1016/ j.ocemod.2008.04.006.

$\longrightarrow$, and $-2008 \mathrm{~b}$ : Towards a mesoscale eddy closure. Ocean Modell., 20, 223-239, doi:10.1016/j.ocemod.2007.09.002.

, and D. Olbers, 2014: An energy compartment model for propagation, nonlinear interaction, and dissipation of internal gravity waves. J. Phys. Oceanogr., 44, 2093-2106, doi:10.1175/ JPO-D-13-0224.1.

-, M. Jochum, and G. Danabasoglu, 2009: Effects of different closures for thickness diffusivity. Ocean Modell., 26, 47-59, doi:10.1016/j.ocemod.2008.08.004.
Emile-Geay, J., and G. Madec, 2009: Geothermal heating, diapycnal mixing and the abyssal circulation. Ocean Sci., 5, 203 217, doi:10.5194/os-5-203-2009.

Ferrari, R., and C. Wunsch, 2009: Ocean circulation kinetic energy: Reservoirs, sources, and sinks. Annu. Rev. Fluid Mech., 41, 253-282, doi:10.1146/annurev.fluid.40.111406.102139.

Ford, R., M. McIntyre, and W. Norton, 2000: Balance and the slow quasimanifold: Some explicit results. J. Atmos. Sci., 57, 1236-1254, doi:10.1175/1520-0469(2000)057<1236:BATSQS>2.0.CO;2.

Ganachaud, A., and C. Wunsch, 2003: Large-scale ocean heat and freshwater transports during the World Ocean Circulation Experiment. J. Climate, 16, 696-705, doi:10.1175/ 1520-0442(2003)016<0696:LSOHAF > 2.0.CO;2.

Gargett, A. E., 1984: Vertical eddy diffusivity in the ocean interior. J. Mar. Res., 42, 359-393, doi:10.1357/002224084788502756.

Gaspar, P., Y. Gregoris, and J.-M. Lefevre, 1990: A simple eddy kinetic energy model for simulations of the oceanic vertical mixing: Tests at station PAPA and Long-Term Upper Ocean Study site. J. Geophys. Res., 95, 16179-16193, doi:10.1029/ JC095iC09p16179.

Gent, P. R., and J. C. McWilliams, 1990: Isopycnal mixing in ocean circulation models. J. Phys. Oceanogr., 20, 150-155, doi:10.1175/1520-0485(1990)020<0150:IMIOCM>2.0.CO;2.

— J. Willebrand, T. J. McDougall, and J. C. McWilliams, 1995: Parameterizing eddy-induced tracer transports in ocean circulation models. J. Phys. Oceanogr., 25, 463-474, doi:10.1175/ 1520-0485(1995)025<0463:PEITTI >2.0.CO;2.

Gregg, M. C., 1989: Scaling turbulent dissipation in the thermocline. J. Geophys. Res., 94, 9686-9698, doi:10.1029/ JC094iC07p09686.

_ _E. A. d'Asaro, T. J. Shay, and N. Larson, 1986: Observations of persistent mixing and near-inertial internal waves. $J$. Phys. Oceanogr., 16, 856-885, doi:10.1175/1520-0485(1986)016<0856: OOPMAN $>2.0 . \mathrm{CO} ; 2$

Griffies, S., and Coauthors, 2009: Coordinated Ocean-Ice Reference Experiments (COREs). Ocean Modell., 26, 1-46, doi:10.1016/j.ocemod.2008.08.007.

Haney, R. L., 1971: Surface thermal boundary condition for ocean circulation models. J. Phys. Oceanogr., 1, 241-248, doi:10.1175/ 1520-0485(1971)001<0241:STBCFO > 2.0.CO;2.

Hasselmann, K., 1968: Weak-interaction theory of ocean waves. Basic Developments in Fluid Dynamics, M. Holt, Ed., Vol. 2, Academic Press, 117-182, doi:10.1016/B978-0-12-395520-3.50008-6.

Held, I., and V. Larichev, 1996: A scaling theory for horizontally homogeneous, baroclinically unstable flow on a beta plane. J. Atmos. Sci., 53, 946-952, doi:10.1175/1520-0469(1996)053<0946: ASTFHH $>2.0 . \mathrm{CO} ; 2$.

Henyey, F., J. Wright, and S. Flatté, 1986: Energy and action flow through the internal wave field: An eikonal approach. J. Geophys. Res., 91, 8487-8495, doi:10.1029/JC091iC07p08487.

Itsweire, E., J. Koseff, D. Briggs, and J. Ferziger, 1993: Turbulence in stratified shear flows: Implications for interpreting shearinduced mixing in the ocean. J. Phys. Oceanogr., 23, 15081522, doi:10.1175/1520-0485(1993)023<1508:TISSFI > 2.0.CO;2.

Jayne, S., 2009: The impact of abyssal mixing parameterizations in an ocean general circulation model. J. Phys. Oceanogr., 39, 1756-1775, doi:10.1175/2009JPO4085.1.

Johnson, D., T. Boyer, H. Garcia, R. Locarnini, O. Baranova, and M. Zweng, 2009: World Ocean Database 2009 documentation. NOAA/NODC Internal Rep. 20, 175 pp.

Kantha, L. H., and C. A. Clayson, 2000: Small Scale Processes in Geophysical Fluid Flows. International Geophysics Series, Vol. 67, Academic Press, 888 pp. 
Key, R. M., and Coauthors, 2004: A global ocean carbon climatology: Results from Global Data Analysis Project (GLODAP). Global Biochem. Cycles, 18, GB4031, doi:10.1029/2004GB002247.

Killworth, P. D., 1997: On the parameterization of eddy transfer. Part I. Theory. J. Mar. Res., 55, 1171-1197, doi:10.1357/ 0022240973224102.

Large, W., G. Danabasoglu, J. McWilliams, P. Gent, and F. Bryan, 2001: Equatorial circulation of a global ocean climate model with anisotropic horizontal viscosity. J. Phys. Oceanogr., 31, 518-536, doi:10.1175/1520-0485(2001)031<0518:ECOAGO>2.0.CO;2.

Larichev, V., and I. Held, 1995: Eddy amplitudes and fluxes in a homogenous model of fully developed baroclinic instability. J. Phys. Oceanogr., 25, 2285-2297, doi:10.1175/ 1520-0485(1995)025<2285:EAAFIA > 2.0.CO;2.

Lorenz, E. N., 1955: Available potential energy and the maintenance of the general circulation. Tellus, 7,157-167, doi:10.1111/ j.2153-3490.1955.tb01148.x.

Lumpkin, R., and K. Speer, 2007: Global ocean meridional overturning. J. Phys. Oceanogr., 37, 2550-2562, doi:10.1175/ JPO3130.1.

Marshall, D. P., and A. J. Adcroft, 2010: Parameterization of ocean eddies: Potential vorticity mixing, energetics and Arnolds first stability theorem. Ocean Modell., 32, 188-204, doi:10.1016/ j.ocemod.2010.02.001

Marshall, J. C., 1981: On the parameterization of geostrophic eddies in the ocean. J. Phys. Oceanogr., 11, 257-271, doi:10.1175/1520-0485(1981)011<0257:OTPOGE >2.0.CO;2.

_ , A. Adcroft, C. Hill, L. Perelman, and C. Heisey, 1997: A finite-volume, incompressible Navier Stokes model for studies of the ocean on parallel computers. J. Geophys. Res., 102, 5753-5766, doi:10.1029/96JC02775.

Matsumoto, K., 2007: Radiocarbon-based circulation age of the world oceans. J. Geophys. Res., 112, C09004, doi:10.1029/ 2007JC004095.

McComas, C., and P. Müller, 1981: Time scales of resonant interactions among oceanic internal waves. J. Phys. Oceanogr., 11, 139-147, doi:10.1175/1520-0485(1981)011<0139: TSORIA $>2.0 . \mathrm{CO} ; 2$.

McDougall, T. J., 2003: Potential enthalpy: A conservative oceanic variable for evaluating heat content and heat fluxes. J. Phys. Oceanogr., 33, 945-963, doi:10.1175/1520-0485(2003)033<0945. PEACOV $>2.0 . \mathrm{CO} ; 2$.

— , and P. C. McIntosh, 2001: The temporal-residual-mean velocity. Part II: Isopycnal interpretation and the tracer and momentum equations. J. Phys. Oceanogr., 31, 1222-1246, doi:10.1175/1520-0485(2001)031<1222:TTRMVP>2.0.CO;2.

Molemaker, M. J., J. C. McWilliams, and I. Yavneh, 2005 Baroclinic instability and loss of balance. J. Phys. Oceanogr., 35, 1505-1517, doi:10.1175/JPO2770.1.

,-- , and X. Capet, 2010: Balanced and unbalanced routes to dissipation in an equilibrated Eady flow. J. Fluid Mech., 654, 35-63, doi:10.1017/S0022112009993272.

Müller, P., G. Holloway, F. Henyey, and N. Pomphrey, 1986: Nonlinear interactions among internal gravity waves. Rev. Geophys., 24, 493-536, doi:10.1029/RG024i003p00493.

Munk, W. H., 1966: Abyssal recipes. Deep-Sea Res. Oceanogr. Abstr., 13, 707-730, doi:10.1016/0011-7471(66)90602-4. , 1981: Internal waves and small-scale processes. Evolution of Physical Oceanography, B. A. Warren and C. Wunsch, Eds., MIT Press, 264-291.

Nikurashin, M., and R. Ferrari, 2011: Global energy conversion rate from geostrophic flows into internal lee waves in the deep ocean. Geophys. Res. Lett., 38, L08610, doi:10.1029/2011GL046576.
Nycander, J., 2011: Energy conversion, mixing energy, and neutral surfaces with a nonlinear equation of state. J. Phys. Oceanogr., 41, 28-41, doi:10.1175/2010JPO4250.1.

Olbers, D. J., 1974: On the Energy Balance of Small-Scale Internal Waves in the Deep Sea. Hamburger Geophysikalische Einzelschriften, Vol. 24, G. M. L. Wittenborn, 91 pp.

- 1976: Nonlinear energy transfer and the energy balance of the internal wave field in the deep ocean. J. Fluid Mech., 74, 375399, doi:10.1017/S0022112076001857.

— , and K. Herterich, 1979: The spectral energy transfer from surface waves to internal waves. J. Fluid Mech., 92, 349-379, doi:10.1017/S0022112079000653.

— , and C. Eden, 2013: A global model for the diapycnal diffusivity induced by internal gravity waves. J. Phys. Oceanogr., 43, 1759-1779, doi:10.1175/JPO-D-12-0207.1.

, J. Willebrand, and C. Eden, 2012: Ocean Dynamics. Springer, 704 pp.

Oort, A. H., S. C. Ascher, S. Levitus, and J. P. Peixóto, 1989: New estimates of the available potential energy in the World Ocean. J. Geophys. Res., 94, 3187-3200, doi:10.1029/ JC094iC03p03187.

Osborn, T. R., 1980: Estimates of the local rate of vertical diffusion from dissipation measurements. J. Phys. Oceanogr., 10, 83-89, doi:10.1175/1520-0485(1980)010<0083:EOTLRO > 2.0.CO;2. , and C. S. Cox, 1972: Oceanic fine structure. Geophys. Astrophys. Fluid Dyn., 3, 321-345, doi:10.1080/03091927208236085.

Pacanowski, R., and S. Philander, 1981: Parameterization of vertical mixing in numerical models of tropical oceans. J. Phys. Oceanogr., 11, 1443-1451, doi:10.1175/1520-0485(1981)011<1443: POVMIN $>2.0 . \mathrm{CO} ; 2$.

Polzin, K. L., J. M. Toole, and R. W. Schmitt, 1995: Finescale parameterizations of turbulent dissipation. J. Phys. Oceanogr., 25, 306-328, doi:10.1175/1520-0485(1995)025<0306: FPOTD $>2.0 . \mathrm{CO} ; 2$.

Rhines, P., 1982: Basic dynamics of the large-scale geostrophic circulation. Summer Study Program in Geophysical Fluid Dynamics, Woods Hole Oceanographic Institution, 1-47.

Rimac, A., J.-S. von Storch, C. Eden, and H. Haak, 2013: The influence of high-resolution wind stress field on the power input to near-inertial motions in the ocean. Geophys. Res. Lett., 40, 4882-4886, doi:10.1002/grl.50929.

Roquet, F., C. Wunsch, and G. Madec, 2011: On the patterns of wind-power input to the ocean circulation. J. Phys. Oceanogr., 41, 2328-2342, doi:10.1175/JPO-D-11-024.1.

Scharffenberg, M. G., and D. Stammer, 2010: Seasonal variations of the large-scale geostrophic flow field and eddy kinetic energy inferred from the TOPEX/Poseidon and Jason-1 tandem mission data. J. Geophys. Res., 115, C02008, doi:10.1029/ 2008JC005242.

Schlösser, F., and C. Eden, 2007: Diagnosing the energy cascade in a model of the North Atlantic. Geophys. Res. Lett., 34, L02604, doi:10.1029/2006GL027813.

Scott, R. B., and F. Wang, 2005: Direct evidence of an oceanic inverse kinetic energy cascade from satellite altimetry. J. Phys. Oceanogr., 35, 1650-1666, doi:10.1175/JPO2771.1.

—, and B. K. Arbic, 2007: Spectral energy fluxes in geostrophic turbulence: Implications for ocean energetics. J. Phys. Oceanogr., 37, 673-688, doi:10.1175/JPO3027.1.

- , and Y. Xu, 2009: An update on the wind power input to the surface geostrophic flow of the world ocean. Deep-Sea Res. I, 56, 295-304, doi:10.1016/j.dsr.2008.09.010.

Sheen, K., and Coauthors, 2013: Rates and mechanisms of turbulent dissipation and mixing in the Southern Ocean: Results 
from the Diapycnal and Isopycnal Mixing Experiment in the Southern Ocean (DIMES). J. Geophys. Res., 118, 2774-2792, doi:10.1002/jgrc.20217.

Smyth, W., J. Moum, and D. Caldwell, 2001: The efficiency of mixing in turbulent patches: Inferences from direct simulations and microstructure observations. J. Phys. Oceanogr., 31, 1969-1992, doi:10.1175/1520-0485(2001)031<1969: TEOMIT $>2.0 . \mathrm{CO} ; 2$.

Starr, V. P., 1968: Physics of Negative Viscosity Phenomena. McGraw-Hill, 256 pp.

Stommel, H., 1982: Is the South Pacific helium-3 plume dynamically active? Earth Planet. Sci. Lett., 61, 63-67, doi:10.1016/ 0012-821X(82)90038-3.

Sun, H., and E. Kunze, 1999: Internal wave-wave interactions. Part II: Spectral energy transfer and turbulence production. J. Phys. Oceanogr., 29, 2905-2919, doi:10.1175/ 1520-0485(1999)029<2905:IWWIPI>2.0.CO;2.

Tailleux, R., 2013: Available potential energy density for a multicomponent Boussinesq fluid with arbitrary nonlinear equation of state. J. Fluid Mech., 735, 499-518, doi:10.1017/jfm.2013.509.

Tandon, A., and C. Garrett, 1996: On a recent parameterization of mesoscale eddies. J. Phys. Oceanogr., 26, 406-416, doi:10.1175/ 1520-0485(1996)026<0406:OARPOM $>2.0 . \mathrm{CO} ; 2$.

Treguier, A. M., I. M. Held, and V. D. Larichev, 1997: Parameterization of quasigeostrophic eddies in primitive equation ocean models. J. Phys. Oceanogr., 27, 567-580, doi:10.1175/ 1520-0485(1997)027<0567:POQEIP > 2.0.CO;2.

Urakawa, L. S., and H. Hasumi, 2010: Role of parameterized eddies in the energy budget of the global thermohaline circulation: Cabbeling versus restratification. J. Phys. Oceanogr., 40, 1894-1901, doi:10.1175/2010JPO4361.1.

, J. A. Saenz, and A. M. Hogg, 2013: Available potential energy gain from mixing due to the nonlinearity of the equation of state in a global ocean model. Geophys. Res. Lett., 40, 2224 2228, doi:10.1002/grl.50508.

Viebahn, J., and C. Eden, 2012: Standing eddies in the meridional overturning circulation. J. Phys. Oceanogr., 42, 1486-1508, doi:10.1175/JPO-D-11-087.1.
Visbeck, M., J. Marshall, T. Haine, and M. Spall, 1997: Specification of eddy transfer coefficients in coarse-resolution ocean circulation models. J. Phys. Oceanogr., 27, 381-402, doi:10.1175/1520-0485(1997)027<0381:SOETCI>2.0.CO;2.

Vollmer, L., and C. Eden, 2013: A global map of meso-scale eddy diffusivities based on linear stability analysis. Ocean Modell., 72, 198-209, doi:10.1016/j.ocemod.2013.09.006.

von Storch, J.-S., H. Sasaki, and J. Marotzke, 2007: Wind-generated power input to the deep ocean: An estimate using a $1 / 10^{\circ}$ general circulation model. J. Phys. Oceanogr., 37, doi:10.1175/ JPO3001.1.

— C. Eden, I. Fast, H. Haak, D. Hernández-Deckers, E. MaierReimer, J. Marotzke, and D. Stammer, 2012: An estimate of the Lorenz energy cycle for the world ocean based on the STORM/NCEP simulation. J. Phys. Oceanogr., 42, 2185-2205, doi:10.1175/JPO-D-12-079.1.

Welander, P., 1973: Lateral friction in the oceans as an effect of potential vorticity mixing. Geophys. Astrophys. Fluid Dyn., 5, 173-189, doi:10.1080/03091927308236114.

Williams, P. D., T. W. Haine, and P. L. Read, 2008: Inertia-gravity waves emitted from balanced flow: Observations, properties, and consequences. J. Atmos. Sci., 65, 3543-3556, doi:10.1175/ 2008JAS2480.1.

Wu, L., Z. Jing, S. Riser, and M. Visbeck, 2011: Seasonal and spatial variations of Southern Ocean diapycnal mixing from Argo profiling floats. Nat. Geosci., 4, 363-366, doi:10.1038/ngeo1156.

Wunsch, C., 1998: The work done by the wind on the oceanic general circulation. J. Phys. Oceanogr., 28, 2332-2340, doi:10.1175/ 1520-0485(1998)028<2332:TWDBTW >2.0.CO;2.

— , and R. Ferrari, 2004: Vertical mixing, energy and the general circulation of the oceans. Annu. Rev. Fluid Mech., 36, 281-314, doi:10.1146/annurev.fluid.36.050802.122121.

Young, W. R., 2010: Dynamic enthalpy, conservative temperature, and the seawater Boussinesq approximation. J. Phys. Oceanogr., 40, 394-400, doi:10.1175/2009JPO4294.1.

Zang, X., and C. Wunsch, 2001: Spectral description of lowfrequency oceanic variability. J. Phys. Oceanogr., 31, 3073-3095, doi:10.1175/1520-0485(2001)031<3073:SDOLFO>2.0.CO;2. 